\title{
On the discrete constitutive models induced by strong discontinuity kinematics and continuum constitutive equations
}

\author{
J. Oliver* \\ E.T.S. Enginyers de Camins, Canals i Ports, Technical University of Catalonia (UPC), Campus Nord UPC (mòdul C-1), Gran Capitán \\ s/n, 08034 Barcelona, Spain
}

Received 21 July 1999; in revised form 27 January 2000

\begin{abstract}
On the basis of continuum constitutive models (stress vs. strain), the introduction of strong discontinuity kinematics (considering jumps in the displacement fields across a discontinuity interface) induces projected discrete constitutive models (traction-displacement jumps) in a consistent manner. Therefore, this projection provides possible links between the classical continuum strain-localization analysis and the non-linear (decohesive) fracture mechanics techniques. The strong discontinuity analysis shows that (bandwidth based) regularization of the hardening/softening parameter is the crucial modification to be done on the continuum model to achieve such a projection, and it also provides the strong discontinuity conditions that set restrictions on the stress state compatible with bifurcations in a strong discontinuity format. The methodology is illustrated on the basis of two classical families of non-linear constitutive models (scalar continuum damage and elasto-plasticity) for which the corresponding discrete constitutive models and the strong discontinuity conditions are derived. (c) 2000 Elsevier Science Ltd. All rights reserved.
\end{abstract}

Keywords: Strain localization; Strong discontinuities; Damage; Plasticity

\section{Introduction}

The analysis and modeling of displacement discontinuities in solid mechanics is a singular problem that can be focused from very different points of view. Linear fracture mechanics has been the classical discipline to tackle that problem although the necessity of getting more deeply involved in the process of formation and propagation of discontinuities led to the development of the non-linear (decohesive) fracture mechanics techniques (Bazant and Planas, 1998) that are essentially based on the introduction of ad hoc discrete constitutive equations (tractions vs. displacement jumps) at one discontinuity interface inside an elastic continuum medium. From the continuum mechanics side, the strain-localization phenomenon (or concentration of the strains in narrow bands) has frequently been envisaged as a way to model displacement

\footnotetext{
${ }^{*}$ Fax: +34-93-401-1048.

E-mail address: oliver@cimne.upc.es (J. Oliver).
} 
discontinuities (when the localization bands become narrower and narrower) on the basis of standard continuum ${ }^{1}$ (stress-strain) non-linear constitutive equations (deBorst et al., 1993).

In recent years, a new methodology to focus that problem based on the concept of strong discontinuity (Simo et al., 1993; Simo and Oliver, 1994; Oliver and Simo, 1994; Oliver, 1995b, 1996a,b, 1998; Armero and Garikipati, 1996; Larsson et al., 1996; Runesson et al., 1996; Oliver et al., 1997-1999; Armero, 1997) that analyzes the onset and development of displacement discontinuities in media governed by standard continuum constitutive equations (stress vs. strain) has been developed. In this context, the discontinuous displacement field induce unbounded strains, and then the so-called strong discontinuity analysis examines the conditions that make these unbounded strains compatible with those continuum constitutive equations while furnishing bounded (and thus having physical meaning) stresses. A typical result in some of those analyses is the derivation of discrete constitutive equations, consistent with their parent continuum constitutive equations, that hold at the interface of discontinuity (Simo et al., 1993; Oliver, 1995a, 1996a; Armero and Garikipati, 1996; Armero, 1997).

This paper extensively deals with this aspect and presents a systematic methodology to derive, from a continuum constitutive model, not only a discrete constitutive equation but also corresponding complete discrete constitutive model. ${ }^{2}$ For that purpose, two target continuum constitutive models, which are frequently considered in the literature on the topic and that represent a wide set of constitutive models, are focused: (1) isotropic scalar continuum damage models and (2) plasticity models.

By means of the strong discontinuity analysis of such models, not only the aforementioned discrete models, but also the so-called strong discontinuity conditions are derived. These non-trivial conditions can
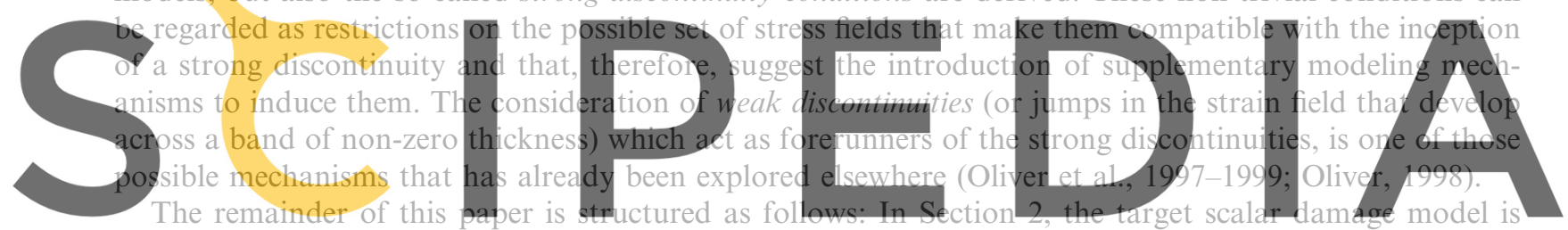

introduced. In Section 3, the original strong discontinuity kinematics as well as a regularized version more

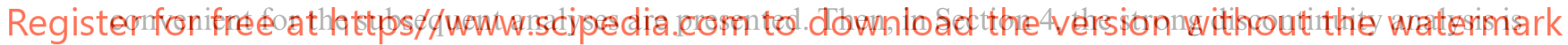
carried out and the corresponding strong discontinuity conditions and discrete constitutive model are obtained. The latter is summarized in Section 5. In Section 6, the standard elasto-plastic (infinitesimal strain based) family of constitutive equations is tackled and the corresponding strong discontinuity analysis is performed in Section 7 and the inherited discrete models are given in Section 8. Finally, in Section 9, some final conclusions are drawn.

\section{Isotropic damage models}

Let us consider the family of isotropic damage models defined by Simó and Ju (1987) and Oliver et al. (1990):

Free energy $\psi(\boldsymbol{\varepsilon}, r)=[1-\mathrm{d}(r)] \psi_{0}(\boldsymbol{\varepsilon}), \quad \psi_{0}=\frac{1}{2} \boldsymbol{\varepsilon}: \mathbf{C}: \boldsymbol{\varepsilon}$,

\footnotetext{
${ }^{1}$ This nomenclature distinguishing discrete constitutive equations (relating tractions to displacement jumps across an interface of discontinuity) and continuum constitutive equations (the ones commonly formulated in continuum mechanics that relate stresses and strains in a continuum medium) will be kept for the rest of this work.

${ }^{2}$ A distinction is made throughout this paper between a constitutive equation, which is understood here as the basic mathematical equation relating stresses (or tractions) and strains (or displacement jumps), and the complete constitutive model in which a constitutive equation is a particular ingredient, that also includes the free energy definition, internal variables, evolution laws, loading-unloading conditions and hardening/softening laws.
} 
Constitutive equation $\quad \boldsymbol{\sigma}=\partial_{\varepsilon} \psi(\boldsymbol{\varepsilon}, r), \quad \boldsymbol{\sigma}=(1-d) \mathbf{C}: \boldsymbol{\varepsilon}$,

Damage variable $\quad d=1-q(r) / r, \quad d \in[0,1]$,

Evolution law $\quad \dot{r}=\lambda, \quad\left\{\begin{array}{l}r \in\left[r_{0}, \infty\right) \\ r_{0}=\left.r\right|_{t=0}=\frac{\sigma_{u}}{\sqrt{E}}\end{array}\right.$

Damage criterion $\quad f(\boldsymbol{\sigma}, q) \equiv \tau_{\sigma}-q, \quad \tau_{\sigma}=\|\boldsymbol{\sigma}\|_{\mathbf{C}^{-1}}=\sqrt{\boldsymbol{\sigma}: \mathbf{C}^{-1}: \boldsymbol{\sigma}}$,

Loading-unloading conditions $\quad f \leqslant 0, \quad \lambda \geqslant 0, \quad \lambda f=0, \quad \lambda \dot{f}=0$ (consistency),

Hardening rule $\dot{q}=\mathscr{H}(r) \dot{r}, \quad\left(\mathscr{H}=q^{\prime}(r) \leqslant 0\right), \quad\left\{\begin{array}{l}q \in\left[0, r_{0}\right], \\ \left.q\right|_{t=0}=r_{0},\end{array}\right.$

where $\psi$ is the free energy, $\varepsilon$ and $\sigma$ are, respectively, the infinitesimal strain and the stress tensors, $r$, the strain-like internal variable, $\psi_{0}$, the elastic free energy and, $\mathbf{C}$, the elasticity tensor defined by $\mathbf{C}=$ $\hat{\lambda} \mathbf{1} \otimes \mathbf{1}+2 \mu \mathbf{I}$ ( $\mathbf{1}$ and $\mathbf{I}$ are the second- and fourth-order unit tensors, respectively, and $\hat{\lambda}$ and $\mu$, the Lamé parameters). Moreover, in Eq. (1a)-(1g), $d$ is the damage variable defined in terms of the hardening/softening variable $q(r)$ which, in turn, evolves in terms of the hardening/softening ${ }^{3}$ parameter $\mathscr{H} ; \lambda \geqslant 0$ is the damage multiplier that appears in the loading-unloading conditions and $f(\sigma, q)$ defines the damage surface

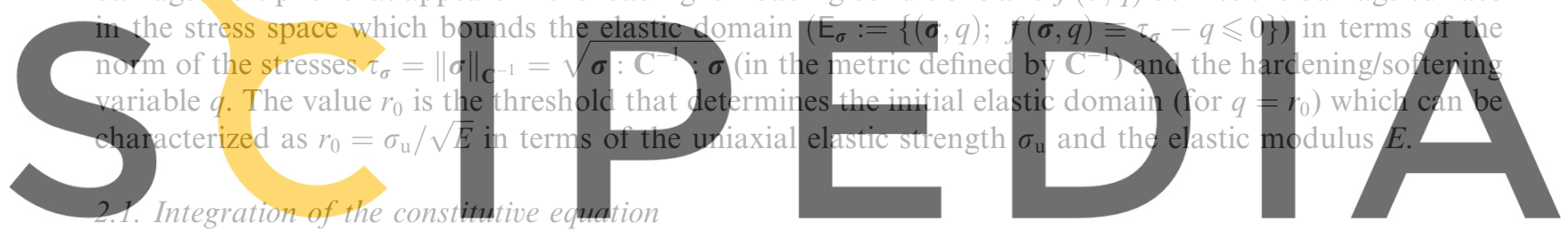

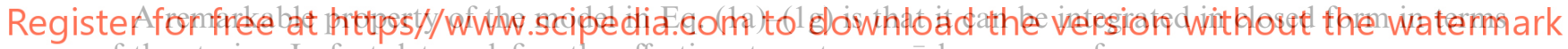
of the strains. In fact, let us define the effective stress tensor $\bar{\sigma}$ by means of

$$
\bar{\sigma}(\varepsilon)=\mathrm{C}: \varepsilon \Rightarrow \sigma=(1-d) \bar{\sigma},
$$

where Eq. (1b) has been taken into account. We now define the following norm in the strain space:

$$
\tau_{\varepsilon} \stackrel{\text { def }}{=}\|\overline{\boldsymbol{\sigma}}(\boldsymbol{\varepsilon})\|_{\mathbf{C}^{-1}}=\sqrt{\overline{\boldsymbol{\sigma}}: \mathbf{C}^{-1}: \overline{\boldsymbol{\sigma}}}=\sqrt{\boldsymbol{\varepsilon}: \mathbf{C}: \boldsymbol{\varepsilon}} \Rightarrow \tau_{\boldsymbol{\sigma}}=(1-d) \tau_{\varepsilon}
$$

where Eq. (2) has been considered. Then, from the damage function $f(\boldsymbol{\sigma}, q)$ and Eq. (1c) $(q=(1-d) r)$, we obtain

$$
f(\boldsymbol{\sigma}, q)=0 \Longleftrightarrow \tau_{\sigma}-q=(1-d) \tau_{\varepsilon}-(1-d) r=0 \Longleftrightarrow g(\varepsilon, r) \equiv \tau_{\varepsilon}-r=0
$$

which states the equivalence of the damage criterion based on $f(\boldsymbol{\sigma}, q)$ with the one based on $g(\boldsymbol{\varepsilon}, r)$. Integration of the internal variable $r$ in Eq. (1d) can now be readily done from Eq. (1f) as

$$
\left.\begin{array}{l}
\dot{r}=\lambda \geqslant 0 \\
\dot{r}>0 \Rightarrow f(\boldsymbol{\sigma}, q)=0 \Longleftrightarrow g(\boldsymbol{\varepsilon}, r)=0 \Longleftrightarrow r=\tau_{\varepsilon} \\
\left.r\right|_{t=0}=r_{0}
\end{array}\right\} \Rightarrow r=\max _{s \in[0, t]}\left(\tau_{\varepsilon}^{t}, r_{0}\right)
$$

\footnotetext{
${ }^{3}$ From now on, it will be assumed that $\mathscr{H} \leqslant 0$ and, thus, that we are dealing with strain softening.
} 
which coincides with the classical result obtained for this family of continuum damage models in terms of the norm $\tau_{\varepsilon}$ (Simó and Ju, 1987; Oliver et al., 1990). Observe in model (1) that once the value of $r$ is determined in terms of the current strains, through Eq. (5), the rest of the variables $(d, \boldsymbol{\sigma})$ can be trivially computed.

Remark 1. As any strain driven rate independent constitutive model the one in Eq. (1) can be regarded as $\boldsymbol{\sigma}(t)=\Sigma\left(\boldsymbol{\varepsilon}_{\tau}\right)$, where $\Sigma$ is a tensorial function of the strain history $\boldsymbol{\varepsilon}_{\tau}$ up to time $t(\tau \in\{0, t\})$, which returns bounded values of the stresses for bounded values of the strains. A similar statement can be made for the rate of the stresses $\dot{\boldsymbol{\sigma}}(t)=\boldsymbol{\Gamma}\left(\boldsymbol{\varepsilon}_{\tau}, \dot{\boldsymbol{\varepsilon}}_{\tau}\right)$, where $\boldsymbol{\Gamma}$ returns bounded values of $\dot{\boldsymbol{\sigma}}$ for bounded values of the strain, $\boldsymbol{\varepsilon}_{\tau}$, and rate of strain, $\dot{\boldsymbol{\varepsilon}}_{\tau}$, histories. These facts will be recalled in subsequent sections.

\section{Kinematics}

The kinematics of a body $\Omega$ exhibiting a discontinuity (jump) of value $\llbracket \dot{\mathbf{u}} \rrbracket(\mathbf{x}, \mathbf{t})$ in the rate of the displacements field (Fig. 1(a) across a material line (for 2D cases) or a material surface (for 3D cases) denoted by $S$, whose normal (pointing to $\Omega^{+}$) is $\mathbf{n}$, can be described as

$$
\dot{\mathbf{u}}(\mathbf{x}, t)=\underbrace{\dot{\overline{\mathbf{u}}}(\mathbf{x}, t)}_{\text {regular (continuous) }}+\underbrace{H_{S}(\mathbf{x}) \llbracket \dot{\mathbf{u}} \rrbracket(\mathbf{x}, t)}_{\text {singular (discontinuous) }}
$$
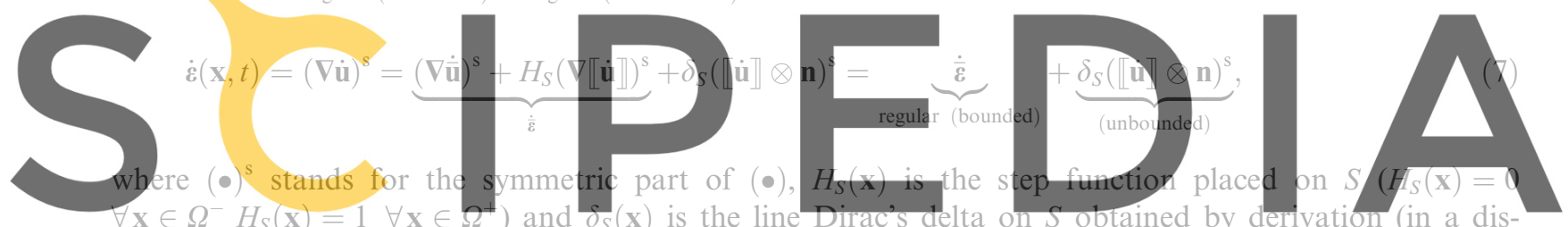

tributional sense) of $H_{S}\left(\nabla H_{S}(\mathbf{x})=\delta_{S} \mathbf{m}\right)$. In Eqs. (6) and (7), (•) stands for the material time derivative (rate)

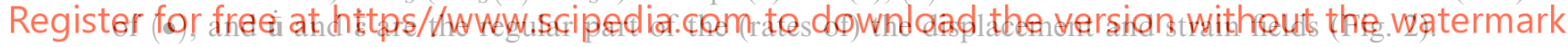

Remark 2. By construction, it is assumed that $\dot{\bar{u}}(\mathbf{x}, t)$ is continuous, and therefore, from Eq. (7), $\dot{\bar{\varepsilon}}(\mathbf{x}, t)$ and $\overline{\mathbf{\varepsilon}}(\mathbf{x}, t)$ exhibit, at most, bounded discontinuities.

A regularized version (more appropriated for our purposes) of the kinematics in Eq. (7) can be obtained by introducing a regularized Dirac's delta function by means of a regularization parameter $h$ and a collocation function $\mu_{S}(\mathbf{x})$ :

$$
\delta_{S}^{h}(\mathbf{x})=\frac{1}{h} \mu_{S}(\mathbf{x}), \quad \mu_{S}(\mathbf{x})= \begin{cases}1, & \mathbf{x} \in S^{h}, \\ 0, & \mathbf{x} \in \Omega \backslash S^{h},\end{cases}
$$

where $S^{h}$ is a discontinuity band, containing $S$, whose width is the regularization parameter $h$ (Fig. 1(b)), in such a way that, in the distributional sense, $\lim _{h \rightarrow 0} \delta_{S}^{h}(\mathbf{x})=\delta_{S}$. Under these assumptions, Eq. (7) reads
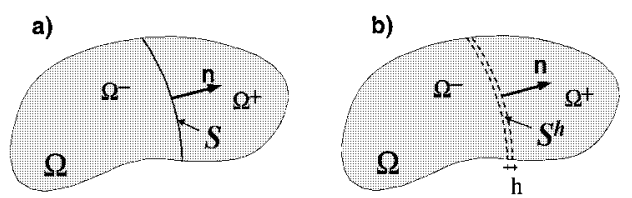

Fig. 1. (a) Discontinuity line $S$ in a body $\Omega$. (b) Regularized discontinuity band $S^{h}$. 

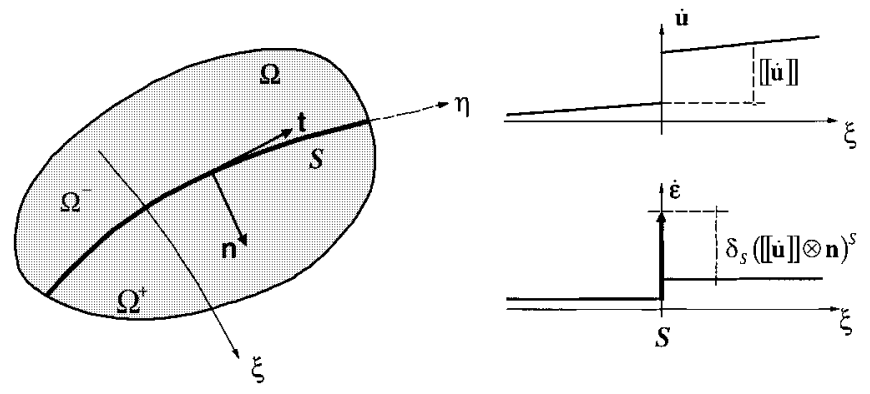

Fig. 2. Strong discontinuity kinematics.

$$
\dot{\varepsilon}^{h}(\mathbf{x}, t)=\dot{\bar{\varepsilon}}+\delta_{S}^{h}(\llbracket \dot{\mathbf{u}} \rrbracket \otimes \mathbf{n})^{\mathrm{s}}=\underbrace{\dot{\bar{\varepsilon}}}_{\text {regular }}+\underbrace{\left.\frac{1}{h} \mu_{S}(\llbracket \dot{\mathbf{u}}] \otimes \mathbf{n}\right)^{\mathrm{s}}}_{\text {unbounded when } h \rightarrow 0}
$$

The kinematics of Eq. (9) allows the introduction of the weak discontinuity concept (Oliver, 1998; Oliver et al., 1998, 1999) for values of the bandwidth $h$ in Eq. (9) different from zero. The jump $\llbracket \mathbf{u} \rrbracket$ then has the meaning of an apparent jump, as the difference of the values of the displacement $\mathbf{u}$ at both sides of the band $S^{h}$ (Fig. 1(b)), and the (rate of ) strain field of Eq. (9) remains discontinuous but bounded. Therefore, when
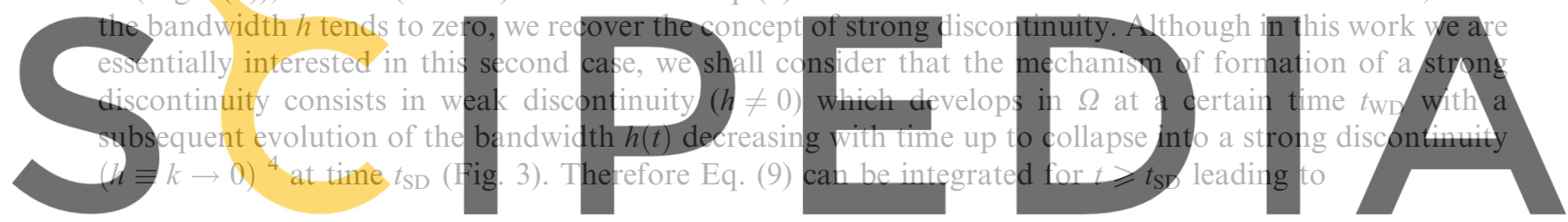

$\left.\left.\left.\varepsilon(\mathbf{x}, t)\right|_{t \geqslant t_{S D}}=\int^{t} \bar{\varepsilon} \mathrm{d} t+\mu_{\varphi} \int^{t_{\mathrm{SD}}} \frac{1}{\bar{t}}(\llbracket \dot{\mathrm{u}}] \otimes n\right)^{\mathrm{s}} \mathrm{d} t+\mu_{\varphi} \int^{t} \frac{1}{\bar{t}}(\llbracket \dot{\mathrm{u}}] \otimes n\right)^{\mathrm{s}} \mathrm{d} t$

Register for free at htes//www.scipedia.com to downlo ad the version without the watermark

$$
=\underbrace{\overline{\overline{\boldsymbol{\varepsilon}}}}_{\text {(bounded for }}+\underbrace{\mu_{S} \frac{1}{h}(\Delta \llbracket \mathbf{u} \rrbracket \otimes \mathbf{n})^{\mathrm{s}}}_{\text {(unbounded for } h \equiv k \rightarrow 0)}
$$

where the material character of $S(\Rightarrow \dot{\mathbf{n}}=0$ ) has been considered. In Eq. (10), $\Delta \llbracket \mathbf{u} \rrbracket$ is the incremental jump (the one developed from the onset of the strong discontinuity at time $t_{\mathrm{SD}}$ to the present time $t>t_{\mathrm{SD}}$ ) i.e.

$$
\Delta \llbracket \mathbf{u} \rrbracket(\mathbf{x}, t) \stackrel{\text { def }}{=} \llbracket \mathbf{u} \rrbracket(\mathbf{x}, t)-\llbracket \mathbf{u} \rrbracket\left(\mathbf{x}, t_{\mathrm{SD}}\right) .
$$

\section{Strong discontinuity analysis}

The strong discontinuity analysis aims at identifying those conditions that make the continuum constitutive model $(1 \mathrm{a}-1 \mathrm{~g})$ compatible with the strong discontinuity kinematics (10). The analysis lies on the traction continuity conditions, across the interface $S$, that emerge from the momentum balance:

\footnotetext{
${ }^{4}$ For computational purposes, the final bandwidth value is set to $h /_{t \geqslant t_{\mathrm{SD}}}=k$, where $k$ is a (very small) regularization parameter.
} 


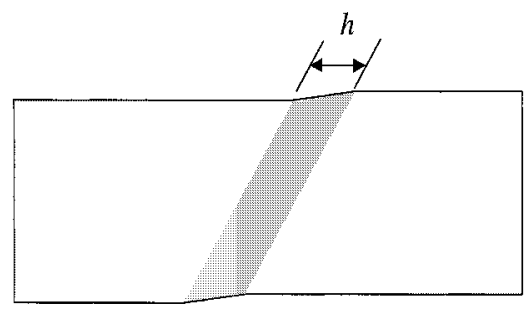

(a)

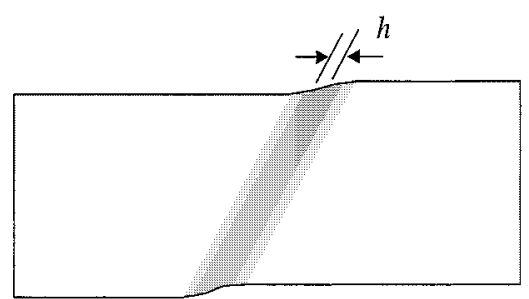

(b)
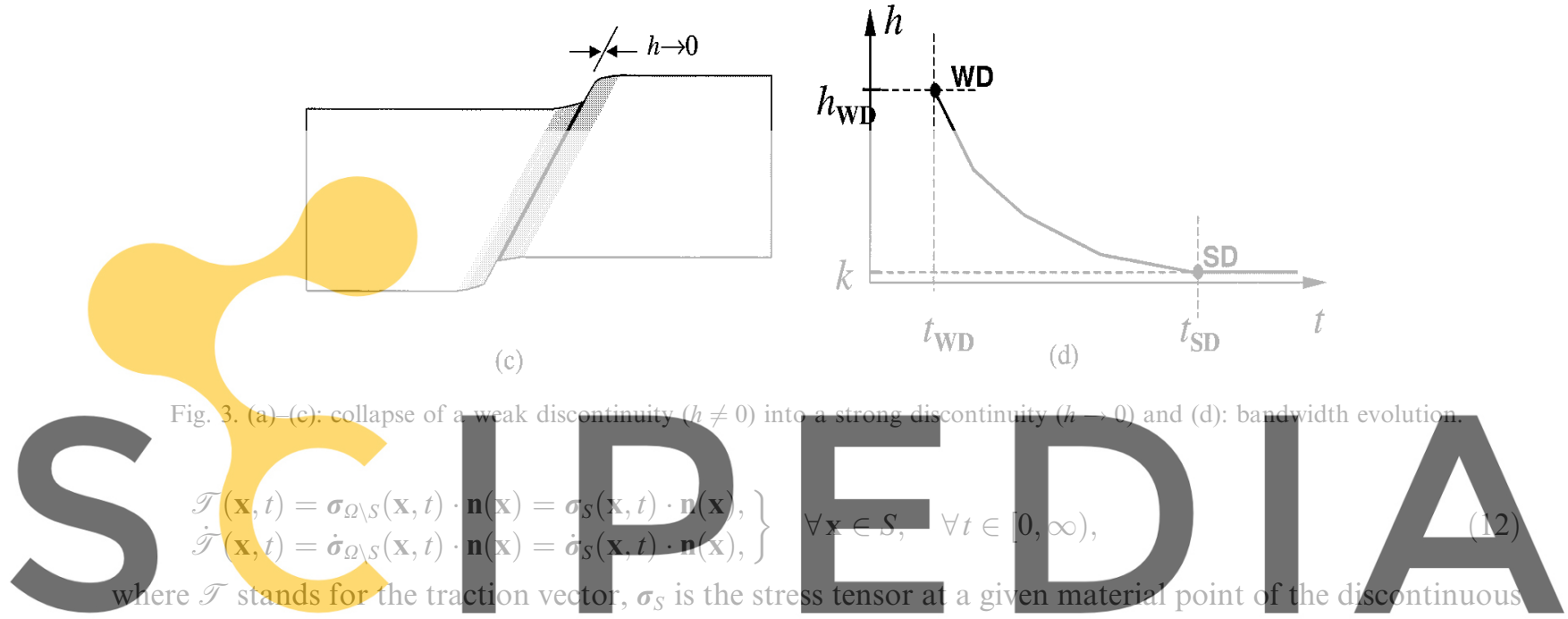

interface $S$ and $\sigma_{\Omega \backslash S}$ are the stresses at a neighbor point on the continuum part of the body $\Omega \backslash S$.

\section{Register for free at https//www.scipedia,com to download the version without the watermark}

- The stresses $\sigma_{\Omega \backslash S}$ are bounded, since the strains $\varepsilon_{\Omega \backslash S}=\bar{\varepsilon}$ are bounded by construction (see Remark 1), and so is $\mathscr{T}=\boldsymbol{\sigma}_{\Omega \backslash S} \cdot \mathbf{n}$ (from Eq. (12)). Therefore, we can also state for any point of the interface $S$ that the product $\mathscr{T}=\boldsymbol{\sigma}_{S} \cdot \mathbf{n}$ is bounded. Then, the three components of $\mathscr{T}$ in the basis formed by the three principal directions (eigenvalues of $\boldsymbol{\sigma}_{S}$ ) read

$$
\begin{aligned}
& \mathscr{T}_{1}=\sigma_{1_{s}} n_{1} \rightarrow(\text { bounded }), \\
& \mathscr{T}_{2}=\sigma_{2_{s}} n_{2} \rightarrow(\text { bounded }), \\
& \mathscr{T}_{3}=\sigma_{3_{s}} n_{3} \rightarrow(\text { bounded }) .
\end{aligned}
$$

- Since the three components of $\mathbf{n}$ are bounded $(\|\mathbf{n}\|=1)$, it follows from Eq. (13) that the three principal stresses $\left(\sigma_{1}, \sigma_{2}, \sigma_{3}\right)_{S}$ are bounded ${ }^{5}$ and, consequently, that every component of the stress tensor $\boldsymbol{\sigma}_{S}$ is bounded despite the strains at $S\left(\varepsilon_{S}\right)$ are unbounded.

- The same reasoning applies to the rate of the stresses and, therefore, $\dot{\sigma}_{S}$ is also bounded despite the strain rates at $S\left(\dot{\boldsymbol{\varepsilon}}_{S}\right)$ are unbounded.

Remark 4. Based on Remark 3 the following statements can also be made:

- The norm $\tau_{\sigma}\left(\boldsymbol{\sigma}_{S}\right)=\left\|\boldsymbol{\sigma}_{S}\right\|_{\mathbf{C}^{-1}}=\sqrt{\boldsymbol{\sigma}_{S}: \mathbf{C}^{-1}: \boldsymbol{\sigma}_{S}}$, in Eq. (1e) is bounded, since $\boldsymbol{\sigma}_{S}$ is bounded,

\footnotetext{
${ }^{5}$ An exception occurs when $n_{i}=0$ for some $i \in\{1,2,3\}$, i.e. when $\mathbf{n}$ is orthogonal to one or two principal stresses. Even in this very particular situation, the bounded character of $\sigma_{S}$ can be shown, in most cases, from alternative reasonings.
} 
- Also, $\dot{\tau}_{\sigma_{S}}\left(=\left(1 / \tau_{\sigma_{S}}\right) \boldsymbol{\sigma}_{S}: \mathbf{C}^{-1}: \dot{\boldsymbol{\sigma}}_{S}\right)$ is bounded, since $\boldsymbol{\sigma}_{S}$ and $\dot{\boldsymbol{\sigma}}_{S}$ are bounded.

- From Eqs. (1d) and (1g) the hardening/softening variable $q_{S}$ evolves only for the loading case $\left(\dot{q}_{S} \neq 0 \Longleftrightarrow \dot{r}=\lambda \neq 0\right)$. In addition, from the consistency condition $\lambda \dot{f}_{S}=0$, in Eq. (1f), we conclude that $\dot{q}_{S} \neq 0 \Rightarrow \lambda \neq 0 \Rightarrow \dot{f}_{S}=0$ and, therefore, $\dot{q}_{S} \neq 0 \Rightarrow \dot{f}_{S}=\dot{\tau}_{\sigma_{S}}-\dot{q}_{S}=0 \Longleftrightarrow \dot{q}_{S}=\dot{\tau}_{\sigma_{S}}$. So, finally, we can state that $\dot{q}_{S} \in\left\{0, \dot{\tau}_{\sigma_{S}}\right\}$ and, consequently, $\dot{q}_{S}$ is bounded.

\subsection{Discrete constitutive equation - Discrete softening law}

Let us consider, at a given point of the interface $S$, the stresses provided by the constitutive model (1) and the strains given by the kinematics in Eq. (10):

$$
\boldsymbol{\sigma}_{S}=\underbrace{(1-d)}_{q\left(r_{S}\right) / r_{S}} \mathbf{C}: \boldsymbol{\varepsilon}_{S}=\frac{q_{S}}{r_{S}} \mathbf{C}:\left[\overline{\boldsymbol{\varepsilon}}+\frac{1}{h}(\Delta \llbracket \mathbf{u} \rrbracket \otimes \mathbf{n})^{\mathrm{s}}\right] .
$$

Now, let us consider the strong discontinuity regime $\left(t>t_{\mathrm{SD}} \Rightarrow h \equiv k \rightarrow 0\right)$ and the tractions $\mathscr{T}$ given by Eq. (14) as

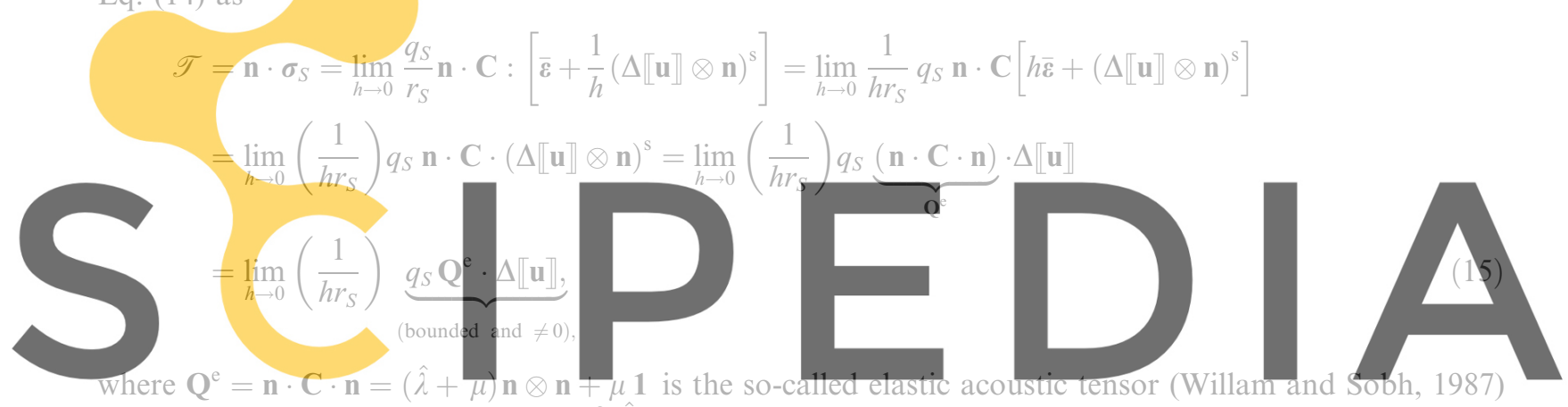

which is positive definite $\left(\operatorname{det}\left(\mathbf{Q}^{\mathrm{e}}\right)=\mu^{2}(\hat{\lambda}+2 \mu)>0\right)$. Observing Eq. (15), we realize that $\mathscr{T}$ is bounded and

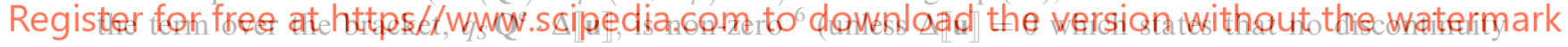

develops). Therefore, the mathematical consistency of Eq. (15) implies that

$$
\lim _{h \rightarrow 0} h r_{S} \neq 0 \quad \text { if } \quad \Delta \llbracket \mathbf{u} \rrbracket \neq 0 .
$$

In order to fulfill such a condition, let us consider the evolution of $r_{S}$ given by

$$
\left.\begin{array}{l}
\dot{r}_{S}=\frac{1}{h} \dot{\bar{\alpha}} \quad \forall t \geqslant t_{\mathrm{WD}}, \\
\left.\bar{\alpha}\right|_{t=t_{\mathrm{WD}}}=0,
\end{array}\right\}
$$

where $\bar{\alpha}$ is a variable that will be imposed to be bounded (as well as its time derivative $\dot{\bar{\alpha}}$ ) which will be named discrete internal variable.

Eq. (17) can be integrated for a given time $t \geqslant t_{\mathrm{SD}}$ (at the strong discontinuity regime) resulting in

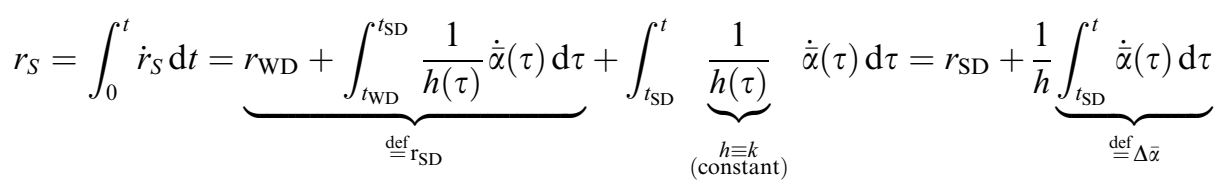

\footnotetext{
${ }^{6}$ Observe that the possibility $\mathbf{Q}^{\mathrm{e}} \cdot \Delta \llbracket \mathbf{u} \rrbracket=0$ and $\Delta \llbracket \mathbf{u} \rrbracket \neq 0$ does not apply since $\mathbf{Q}^{\mathrm{e}}$ is non-singular.
} 


$$
\Rightarrow\left\{\begin{array}{l}
r_{S}=r_{\mathrm{SD}}+\frac{1}{h} \Delta \bar{\alpha}, \\
r_{\mathrm{SD}}=\left.r_{S}\right|_{t=t_{\mathrm{SD}}}=r_{\mathrm{WD}}+\int_{t_{\mathrm{WD}}}^{t_{\mathrm{SD}}} \frac{1}{h(\tau)} \dot{\bar{\alpha}}(\tau) \mathrm{d} \tau, \\
r_{\mathrm{WD}}=\left.r_{S}\right|_{t=t_{\mathrm{WD}}}, \\
\Delta \bar{\alpha}_{t}=\bar{\alpha}_{t}-\bar{\alpha}_{t_{\mathrm{SD}}} \in[0, \infty) .
\end{array}\right.
$$

The term $r_{\mathrm{SD}}$ in Eq. (19) fulfills (for $t \geqslant t_{\mathrm{SD}}$ ):

$$
\lim _{h \rightarrow 0} h r_{\mathrm{SD}}=\lim _{k \rightarrow 0} k r_{\mathrm{SD}}=\lim _{k \rightarrow 0}\left[k r_{\mathrm{WD}}+\int_{t_{\mathrm{WD}}}^{t_{\mathrm{SD}}} \frac{k}{h(\tau)} \dot{\bar{\alpha}}(\tau) \mathrm{d} \tau\right]=0
$$

and, therefore, substitution of Eqs. (19) and (20) into Eq. (16) yields

$$
\lim _{h \rightarrow 0} h r_{S}=\lim _{h \rightarrow 0}\left(h r_{\mathrm{SD}}+h \frac{1}{h} \Delta \bar{\alpha}\right)=\Delta \bar{\alpha} \neq 0
$$

which proves that the evolution law (17) is consistent with condition (16) and makes Eq. (15) compatible for $\Delta \llbracket \mathbf{u} \rrbracket \neq 0$.

Now, by substituting Eq. (21) into Eq. (15), we obtain

Discrete constitutive equation $\rightarrow \mathscr{T}=\frac{q_{S}}{\Delta \bar{\alpha}} \mathbf{Q}^{\mathrm{e}} \cdot \Delta \llbracket \mathbf{u} \rrbracket \quad \forall t \geqslant t_{\mathrm{SD}}$

which constitutes the discrete constitutive equation that furnishes the tractions $\mathscr{T}$ on the interface $S$ in terms

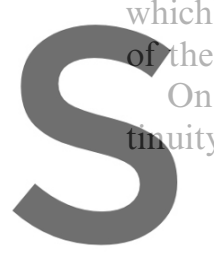

On the other hand, by

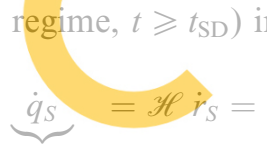

(bounded)
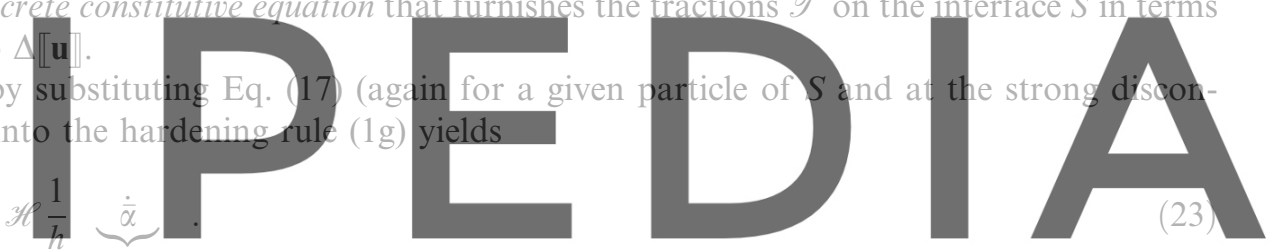

Register for free at https//www.scipedia.com to download the version without the watermark

rm $\mathscr{H} 1 / h$ in Eq. (23) has to be bounded at the strong discontinuity regime, i.e.

$$
\lim _{h \rightarrow 0} \mathscr{H} \frac{1}{h}=\text { bounded }=\overline{\mathscr{H}}
$$

where the bounded parameter $\overline{\mathscr{H}}<0$ will be termed discrete softening parameter to be distinguished from the continuum one, $\mathscr{H}$, that appears in the constitutive model (1g). In order to fulfill condition (24), we shall impose

$$
\mathscr{H}(t)=h(t) \overline{\mathscr{H}} \quad \forall t \geqslant t_{\mathrm{WD}} .
$$

Remark 5. When applied to the strong discontinuity regime $(h \equiv k \rightarrow 0) \mathrm{Eq}$. (25) is the so-called softening regularization condition (Simo et al., 1993; Simo and Oliver, 1994; Oliver, 1996a; Armero and Garikipati, 1996). Here, that condition is extended to the weak discontinuity regime in the context of the variable bandwidth model referred to in Section 3 and Fig. 3.

Finally, by substituting Eq. (25) into Eq. (23), the discrete softening law emerges as

$$
\dot{q}_{S}(t)=\overline{\mathscr{H}} \dot{\bar{\alpha}}, \quad q_{S} \in\left[0, r_{0}\right] \quad \forall t \geqslant t_{\mathrm{SD}}
$$

which can be integrated along time leading to 


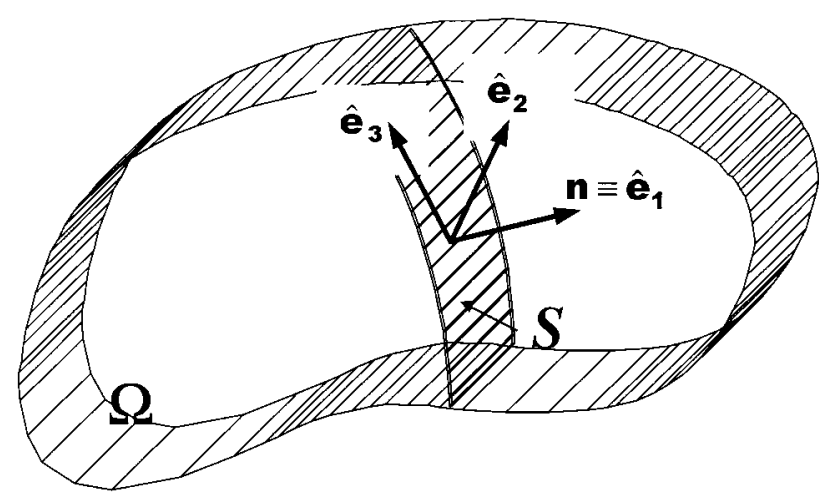

Fig. 4. Local reference system at the discontinuity interface $S$.

\section{Discrete softening law $\rightarrow \bar{q}(\Delta \bar{\alpha})=q_{S}=\underbrace{\left.q_{S}\right|_{t=t_{\mathrm{SD}}}}_{q_{\mathrm{SD}}}+\int_{t_{\mathrm{SD}}}^{t} \overline{\mathscr{H}} \dot{\bar{\alpha}}(\tau) \mathrm{d} \tau=q_{\mathrm{SD}}+\overline{\mathscr{H}} \Delta \bar{\alpha} \nless 0$}

which states the direct dependence ${ }^{7}$ of the hardening/softening variable at the interface $\left(q_{S}\right)$ on the discrete internal variable $\Delta \bar{\alpha}$ through the discrete softening parameter $\overline{\mathscr{H}}$. On the basis of this result, the discrete
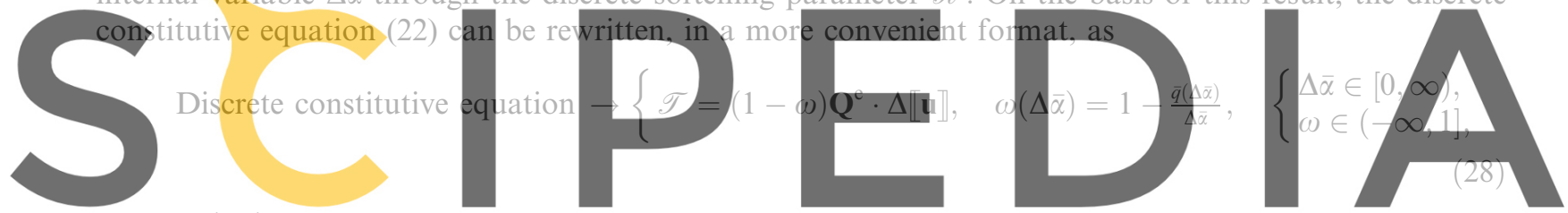

where $\omega(\Delta \bar{\alpha})$ can be understood as the discrete damage variable ranging from $\omega=-\infty$ (for $\Delta \bar{\alpha}=0$ at the

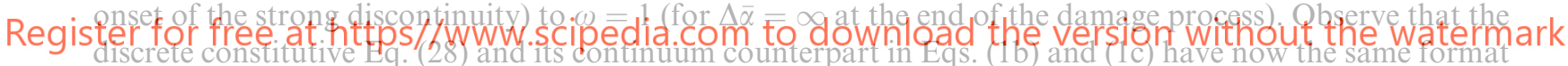
and that there is the following (one to one) correspondence between the continuum and discrete variables:

\begin{tabular}{||l||c|c|c|c|c||c||}
\hline \hline Continuum: & $\boldsymbol{\sigma}$ & $\boldsymbol{\varepsilon}$ & $\mathbf{C}$ & $d$ & $r$ & $q(r)$ \\
\hline Discrete: & $\mathcal{T}$ & $\Delta \llbracket \mathbf{u} \rrbracket$ & $\mathbf{Q}^{e}=\mathbf{n} \cdot \mathbf{C} \cdot \mathbf{n}$ & $\omega$ & $\Delta \bar{\alpha}$ & $\bar{q}(\Delta \bar{\alpha})$ \\
\hline \hline
\end{tabular}

Let us now consider a local orthonormal base $\left(\hat{\mathbf{e}}_{1}, \hat{\mathbf{e}}_{2}, \hat{\mathbf{e}}_{3}\right)$, where the first element coincides with the normal vector $\left(\hat{\mathbf{e}}_{1} \equiv \mathbf{n}\right.$ ) (Fig. 4). Considering the isotropic elastic constitutive tensor $\mathbf{C}$, defined in Section 1 $(\mathbf{C}=\hat{\lambda} \mathbf{1} \otimes \mathbf{1}+2 \mu \mathbf{I})$, the elastic acoustic tensor $\mathbf{Q}^{\mathrm{e}}$ reads

$$
\mathbf{Q}^{\mathrm{e}}=\mathbf{n} \cdot \mathbf{C} \cdot \mathbf{n}=(\hat{\lambda}+2 \mu) \hat{\mathbf{e}}_{1} \otimes \hat{\mathbf{e}}_{1}+\mu \hat{\mathbf{e}}_{2} \otimes \hat{\mathbf{e}}_{2}+\mu \hat{\mathbf{e}}_{3} \otimes \hat{\mathbf{e}}_{3} .
$$

\footnotetext{
${ }^{7}$ Here, this dependence becomes linear since, for the sake of simplicity, a constant value for $\overline{\mathscr{H}}$ has been considered. However, there is no restriction to extend this case to a non-constant discrete softening parameter $\overline{\mathscr{H}}(\bar{\alpha})$.
} 


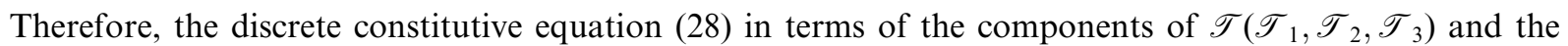
components of $\Delta \llbracket \mathbf{u} \rrbracket\left(\Delta \llbracket u \rrbracket_{1}, \Delta \llbracket u \rrbracket_{2}, \Delta \llbracket u \rrbracket_{3}\right)$ reads

$$
\begin{aligned}
& \mathscr{T}_{1}=(1-\omega)(\hat{\lambda}+2 \mu) \Delta \llbracket u \rrbracket_{1}, \\
& \mathscr{T}_{2}=(1-\omega) \mu \Delta \llbracket u \rrbracket_{2}, \\
& \mathscr{T}_{3}=(1-\omega) \mu \Delta \llbracket u \rrbracket_{3} .
\end{aligned}
$$

\subsection{Discrete damage free energy}

Let us now focus on the continuum free energy of Eq. (1a) for a given particle of $S$ :

$$
\left.\psi(\boldsymbol{\varepsilon}, r)\right|_{\mathbf{x} \in S}=\psi\left(\boldsymbol{\varepsilon}_{S}, r_{S}\right)=\psi(\underbrace{\overline{\boldsymbol{\varepsilon}}+\frac{1}{h}(\Delta \llbracket \mathbf{u} \rrbracket \otimes \mathbf{n})^{\mathrm{s}}}, r_{S})=\hat{\psi}\left(\overline{\boldsymbol{\varepsilon}}, \Delta \llbracket \mathbf{u} \rrbracket, r_{S}\right),
$$

where the discontinuity kinematics of Eq. (10) has been considered. Considering Eqs. (1a) and (1c), Eq. (32) can be rewritten as

$$
\hat{\psi}\left(\bar{\varepsilon}, \Delta \llbracket \mathbf{u} \rrbracket, r_{S}\right)=\underbrace{(1-d)}_{q\left(r_{S}\right) / r_{S}}\left(r_{S}\right) / r_{S} \underbrace{\psi_{0}\left(\bar{\varepsilon}+\frac{1}{h}(\Delta \llbracket \mathbf{u} \rrbracket \otimes \mathbf{n})^{S}\right)}=\frac{q_{S}}{r_{S}} \hat{\psi}_{0}
$$
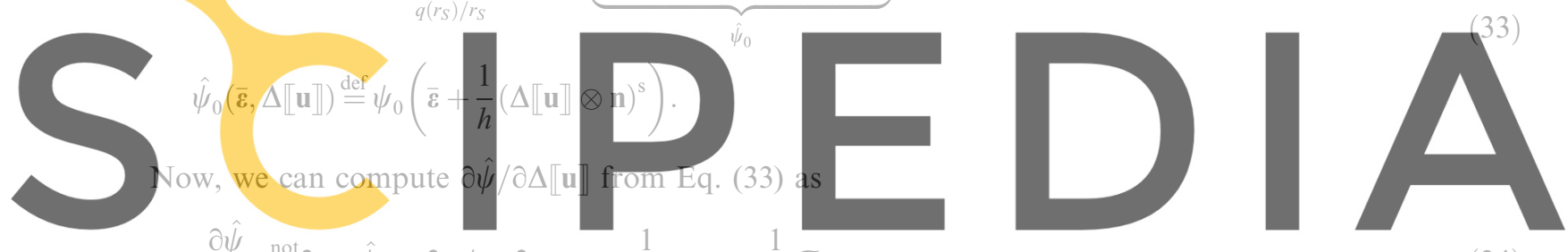

Register for free at https $/ /_{\sigma_{S}} w w_{1, S C i p}$ pedia.com to download the version without the watermark

where Eqs. (1b) $\left(\sigma=\partial_{\varepsilon} \psi\right)$ and (10) have been taken into account and $\left[(1 \otimes \mathrm{n})^{\mathrm{s}}\right]_{i j k} \stackrel{\text { def }}{=} \frac{1}{2}\left(\delta_{i k} n_{j}+\delta_{j k} n_{i}\right)$.

Therefore, from Eq. (34), we obtain

$$
\mathscr{T}=h \partial_{\Delta \llbracket \mathbf{u} \rrbracket} \hat{\psi}\left(\overline{\boldsymbol{\varepsilon}}, \Delta \llbracket \mathbf{u} \rrbracket, r_{S}\right) .
$$

Let us now consider the strong discontinuity regime (for $t \geqslant t_{\mathrm{SD}}$ ) and, thus, $h \equiv k=$ constant. Then, Eq. (35) reads

$$
\mathscr{T}=\lim _{h \rightarrow 0} h \partial_{\Delta \llbracket \mathbf{u} \rrbracket} \hat{\psi}=\partial_{\Delta \llbracket \mathbf{u} \rrbracket} \underbrace{\left[\lim _{h \rightarrow 0}(h \hat{\psi})\right]}_{\varphi}=\partial_{\Delta \llbracket \mathbf{u} \rrbracket} \varphi, \quad \varphi \stackrel{\text { def }}{=} \lim _{h \rightarrow 0}(h \hat{\psi})=\lim _{h \rightarrow 0}\left(h \psi\left(\boldsymbol{\varepsilon}_{S}, r_{S}\right)\right),
$$

where the discrete damage free energy $\varphi=\lim _{h \rightarrow 0}\left(h \psi\left(\varepsilon_{S}, r_{S}\right)\right)$ can be immediately identified as the free energy per unit surface ${ }^{8}$ of discontinuity interface $S$. The explicit expression of $\varphi$ can be then straightforwardly obtained from Eqs. (1a), (33) and (21), after some trivial algebraic operations, as

\footnotetext{
${ }^{8}$ Since $\psi$ is the free energy per unit volume (free energy density), considering an elemental volume $\mathrm{d} V$ at the discontinuity interface (corresponding to a differential discontinuity surface $\mathrm{d} S$ and having a bandwidth $h$ ), one can write $\mathrm{d} V=h \mathrm{~d} S$. Thus, the free energy associated to this differential volume is $\mathrm{d} \psi=h \mathrm{~d} V=h \psi \mathrm{d} S$ and the free energy per unit of discontinuity surface results in $\varphi=\mathrm{d} \psi / \mathrm{d} S=h \psi$.
} 
Discrete damage free energy $\rightarrow\left\{\begin{array}{l}\varphi(\omega, \Delta \llbracket \mathbf{u} \rrbracket)=(1-\omega) \varphi_{0}(\Delta \llbracket \mathbf{u} \rrbracket), \\ \varphi_{0}(\Delta \llbracket \mathbf{u} \rrbracket)=\frac{1}{2} \Delta \llbracket \mathbf{u} \rrbracket \cdot \mathbf{Q}^{\mathrm{e}} \cdot \Delta \llbracket \mathbf{u} \rrbracket .\end{array}\right.$

Observe again that the discrete free energy $\varphi$ of Eq. (37) and the continuum free energy $\psi$ of Eq. (1a) exhibit the same format in terms of the corresponding variables in Eq. (29).

\subsection{Strong discontinuity conditions}

In the previous sections, only the bounded character of the traction $\mathscr{T}$ has been exploited. This is what was (implicitly or explicitly) done in the pioneering strong discontinuity analyses (Simo et al., 1993; Oliver, 1996a; Armero and Garikipati, 1996). However, as it was stated in Remark 3, not only $\mathscr{T}$, but also the entire stress tensor at the discontinuous interface $\sigma_{S}$ remains bounded at the strong discontinuity regime, and this fact is now being exploited. So, let us consider the stress field $\sigma_{S}$ supplied by the continuum constitutive equation (1b) and (1c) at a certain point of $S$ :

$$
\boldsymbol{\sigma}_{S}=(1-d) \mathbf{C}: \boldsymbol{\varepsilon}_{S}=\frac{q\left(r_{S}\right)}{r_{S}} \mathbf{C}: \boldsymbol{\varepsilon}_{S}
$$

Inserting the kinematics (10) and considering the strong discontinuity regime $\left(t \geqslant t_{\mathrm{SD}}\right.$ and, thus, $\left.h \rightarrow 0\right)$,

$$
\begin{aligned}
\boldsymbol{\sigma}_{S} & =\lim _{h \rightarrow 0} \frac{\bar{q}}{r_{\mathrm{SD}}+\frac{1}{h} \Delta \bar{\alpha}} \mathbf{C}:\left[\overline{\boldsymbol{\varepsilon}}+\frac{1}{h}(\Delta \llbracket \mathbf{u} \rrbracket \otimes \mathbf{n})^{\mathrm{s}}\right]=\lim _{h \rightarrow 0} \frac{\bar{q}}{h r_{\mathrm{SD}}+\Delta \bar{\alpha}} \mathbf{C}:\left[h \overline{\boldsymbol{\varepsilon}}+(\Delta \llbracket \mathbf{u} \rrbracket \otimes \mathbf{n})^{\mathrm{s}}\right] \\
& =\frac{\bar{q}}{\Delta \bar{\alpha}} \mathbf{C}:(\Delta \llbracket \mathbf{u} \rrbracket \otimes \mathbf{n})^{\mathrm{s}},
\end{aligned}
$$

where Eqs. (19) and (20) have been taken into account. Now, Eq. (39) can be rewritten as

$$
(\Delta \llbracket \mathbf{u} \rrbracket \otimes \mathbf{n})^{\mathrm{s}}=\frac{\Delta \bar{\alpha}}{\bar{q}} \underbrace{\mathbf{C}^{-1}: \boldsymbol{\sigma}_{S}}_{\bar{\varepsilon}_{S}}=\frac{\Delta \bar{\alpha}}{\bar{q}} \overline{\boldsymbol{\varepsilon}}_{S},
$$

where the effective strain field $\overline{\boldsymbol{\varepsilon}}\left(\overline{\boldsymbol{\varepsilon}}_{\mathrm{def}}^{=} \mathbf{C}^{-1}: \boldsymbol{\sigma}=(1-d) \boldsymbol{\varepsilon}\right)$ has been considered. Eq. (40) is the so-called strong discontinuity equation (Oliver, 1996a; Oliver et al., 1997-1999) which provides a relationship between the stresses $\boldsymbol{\sigma}_{S}$ and the displacement jump $\Delta \llbracket \mathbf{u} \rrbracket$ which has to be fulfilled for all $t \geqslant t_{\mathrm{SD}}$. That tensorial equation can be regarded as a set of six (due to symmetry) algebraic equations relating $\sigma_{S}$ and $\Delta \llbracket \mathbf{u} \rrbracket$. Three of them are in fact the discrete constitutive equations (28) or (31) (which can be extracted multiplying by $\mathbf{n}$, both sides of Eq. (40) and performing some trivial algebraic manipulations). Apart from them, three additional equations (now relating only components of $\boldsymbol{\sigma}_{S}$ ) can be obtained from that equation. They can be directly derived observing the components of Eq. (40) in the aforementioned orthonormal base ( $\left.\hat{\mathbf{e}}_{1} \equiv \mathbf{n}, \hat{\mathbf{e}}_{2}, \hat{\mathbf{e}}_{3}\right)$ :

$$
\left[\begin{array}{ccc}
\Delta \llbracket u \rrbracket_{1} & \frac{1}{2} \Delta \llbracket u \rrbracket_{2} & \frac{1}{2} \Delta \llbracket u \rrbracket_{3} \\
\frac{1}{2} \Delta \llbracket u \rrbracket_{2} & 0 & 0 \\
\frac{1}{2} \Delta \llbracket u \rrbracket_{3} & 0 & 0
\end{array}\right]=\frac{\Delta \bar{\alpha}}{\bar{q}}\left[\begin{array}{lll}
\bar{\varepsilon}_{11} & \bar{\varepsilon}_{12} & \bar{\varepsilon}_{13} \\
\bar{\varepsilon}_{12} & \bar{\varepsilon}_{22} & \bar{\varepsilon}_{23} \\
\bar{\varepsilon}_{13} & \bar{\varepsilon}_{23} & \bar{\varepsilon}_{33}
\end{array}\right]_{S}
$$

and realizing that components $(\bullet)_{22},(\bullet)_{23}$ and $(\bullet)_{23}$ in Eq. (41) lead to

$$
\begin{aligned}
\text { Strong discontinuity conditions } & \rightarrow \bar{\varepsilon}_{22_{S}}=\bar{\varepsilon}_{23_{S}}=\bar{\varepsilon}_{33_{S}}=0 \Rightarrow\left[\mathbf{C}^{-1}: \boldsymbol{\sigma}_{S}\right]_{22}=\left[\mathbf{C}^{-1}: \boldsymbol{\sigma}_{S}\right]_{23} \\
& =\left[\mathbf{C}^{-1}: \boldsymbol{\sigma}_{S}\right]_{33}=0 .
\end{aligned}
$$

Remark 6. Conditions (42) constitute the strong discontinuity conditions (Oliver, 1996a; Oliver et al., 19971999) which set crucial restrictions on the stress state at the inception of the strong discontinuity. In fact, these conditions (eventually on the stresses) will not be fulfilled, in general, at the bifurcation of the stress-strain 
fields which precedes the formation of the discontinuity (Runesson et al., 1991; Ottosen and Runesson, 1991). Therefore, they preclude that such bifurcation takes place by inception of a strong discontinuity $(h \rightarrow 0)$ and, at most, a weak discontinuity $(h \neq n)$ is induced. In consequence, a procedure to enforce the strong discontinuity conditions (42) should be included in models aiming at the simulation of strong discontinuities (Oliver et al., 1999).

Remark 7. The role of the strong discontinuity conditions (42) can be examined from a different point of view. Substitution of the discrete constitutive Eq. (22) into Eq. (39) yields

$$
\boldsymbol{\sigma}_{S}=\frac{\bar{q}}{\Delta \bar{\alpha}} \mathbf{C}:(\Delta \llbracket \mathbf{u} \rrbracket \otimes \mathbf{n})^{\mathrm{s}}=\frac{\bar{q}}{\Delta \bar{\alpha}}(\mathbf{C} \cdot \mathbf{n}) \cdot \Delta \llbracket \mathbf{u} \rrbracket=\underbrace{(\mathbf{C} \cdot \mathbf{n}) \cdot\left[\mathbf{Q}^{\mathrm{e}}\right]^{-1}}_{\mathbf{M}} \cdot \mathscr{T}=\mathbf{M} \cdot \mathscr{T} \quad \forall t \geqslant t_{\mathrm{SD}}
$$

which states that, at the strong discontinuity regime, the stress state at the discontinuity interface $\boldsymbol{\sigma}_{S}$ is fully determined in terms of the traction vector ${ }^{9} \mathscr{T}\left(\boldsymbol{\sigma}_{S}(\mathscr{T})=\mathbf{M}(\mathbf{n}) \cdot \mathscr{T}\right)$. Eq. (43) constitute a system of six (due to the symmetry of $\left.\boldsymbol{\sigma}_{S}\right)$ equations from which the three components of the traction vector $\mathscr{T}$ can be eliminated. The remaining three equations, now involving only components of $\boldsymbol{\sigma}_{S}$, are the strong discontinuity conditions (42). In other words, the strong discontinuity conditions guarantee consistency in Eq. (43) and, thus, that the stress state $\boldsymbol{\sigma}_{S}$ at the interface is fully determined by solely the traction vector $\mathscr{T}$.

\subsection{Discrete norms - damage criteria}

The particular stress-state (43) induced by the strong discontinuity conditions (42) also affects the structure of the continuum norm $\tau_{\sigma}=\|\boldsymbol{\sigma}\|_{\mathbf{C}^{-1}}$ in Eq. (1e). In fact, from Eq. (39) we obtain

$$
\begin{aligned}
\tau_{\boldsymbol{\sigma}_{S}} & =\sqrt{\boldsymbol{\sigma}_{S}: \mathbf{C}^{-\mathbf{1}}: \sigma_{S}}=\left[\left(\frac{\bar{q}}{\Delta \bar{\alpha}}\right)^{2}(\Delta \llbracket \mathbf{u} \rrbracket \otimes \mathbf{n})^{\mathrm{s}}: \mathbf{C}:(\Delta \llbracket \mathbf{u} \rrbracket \otimes \mathbf{n})^{\mathrm{s}}\right]^{1 / 2}=\frac{\bar{q}}{\Delta \bar{\alpha}}(\underbrace{\mathbf{n} \cdot \mathbf{C} \cdot \mathbf{n}]}_{\mathbf{Q}^{\mathrm{e}}} \cdot \Delta \llbracket \mathbf{u} \rrbracket)^{1 / 2} \\
& =\frac{\bar{q}}{\Delta \bar{\alpha}} \sqrt{\Delta \llbracket \mathbf{u} \rrbracket \cdot \mathbf{Q}^{\mathrm{e}} \cdot \Delta \llbracket \mathbf{u} \rrbracket},
\end{aligned}
$$

and substituting the discrete constitutive equation (22) $\left(\Delta \llbracket \mathbf{u} \rrbracket=\frac{\Delta \bar{\alpha}}{\bar{q}}\left[\mathbf{Q}^{\mathrm{e}}\right]^{-1} \cdot \mathscr{T}\right)$ into Eq. (44), one gets

$$
\tau_{\sigma_{S}}=\sqrt{\mathscr{T} \cdot\left[\mathbf{Q}^{\mathrm{e}}\right]^{-1} \cdot \mathscr{T}}=\|\mathscr{T}\|_{\left[\mathbf{Q}^{\mathrm{e}}\right]^{-1}} \stackrel{\text { def }}{=} \tau_{\mathscr{T}} .
$$

Therefore, due to the strong discontinuity conditions (42), the continuum norm of the stresses $\tau_{\boldsymbol{\sigma}}=\|\boldsymbol{\sigma}\|_{\mathbf{C}^{-1}}$, in the metric $\mathbf{C}^{-1}$, is projected onto the discontinuity interface $S$ as a discrete norm of the tractions $\tau_{\mathscr{T}}=\|\mathscr{T}\|_{\left[\mathbf{Q}^{\mathrm{e}}\right]^{-1}}$ in the metric $\left[\mathbf{Q}^{\mathrm{e}}\right]^{-1}$. Observe again the agreement with the correspondence table (29).

Finally, a discrete norm of the displacement jump $\tau_{\llbracket \Delta \mathbf{u} \rrbracket}$ can be defined at $S$ as

$$
\tau_{\llbracket \Delta \mathbf{u} \rrbracket}=\sqrt{\Delta \llbracket \mathbf{u} \rrbracket \cdot \mathbf{Q}^{\mathrm{e}} \cdot \Delta \llbracket \mathbf{u} \rrbracket}=\|\Delta \llbracket \mathbf{u} \rrbracket\|_{\mathbf{Q}^{\mathrm{e}}}
$$

and, from Eqs. (44), (46) and (28) one can write

$$
\tau_{\mathscr{T}}=\frac{\bar{q}}{\Delta \bar{\alpha}} \tau_{\Delta \llbracket \mathbf{u} \rrbracket}=(1-\omega) \tau_{\Delta \llbracket \mathbf{u} \rrbracket}
$$

which constitutes the discrete counterpart of relationship (3) that holds for the continuum norms $\tau_{\sigma}$ and $\tau_{\varepsilon}$. Finally, the damage criterion (1e) translates, from Eq. (45) into

\footnotetext{
${ }^{9}$ Therefore, from $\mathscr{T}=\sigma_{\Omega \backslash S} \cdot \mathbf{n}, \boldsymbol{\sigma}_{S}$ is fully determined in terms of the stresses $\boldsymbol{\sigma}_{\Omega \backslash S}$ (at the continuous neighborhood of $S$ ).
} 


$$
f\left(\boldsymbol{\sigma}_{S}, q\right) \equiv \tau_{\boldsymbol{\sigma}_{S}}-q \equiv \tau_{\mathscr{T}}(\mathscr{T})-\bar{q} \stackrel{\text { def }}{=} \mathscr{F}(\mathscr{T}, \bar{q})
$$

and the loading-unloading conditions (1f) read

$$
\mathscr{F} \leqslant 0, \quad \bar{\lambda} \geqslant 0, \quad \bar{\lambda} \mathscr{F}=0, \quad \bar{\lambda} \dot{\mathscr{F}}=0,
$$

where the discrete damage multiplier $\bar{\lambda}$ emerges from the discrete evolution laws (17), (19) and (1d):

$$
\frac{\partial}{\partial t}(\Delta \bar{\alpha})=\dot{\bar{\alpha}}=h \dot{r}_{S}=h \lambda_{S} \stackrel{\text { def }}{=} \bar{\lambda} \geqslant 0 .
$$

\section{Discrete damage model}

In view of the strong discontinuity analysis in Section 4, a discrete constitutive model at the interface $S$, emerging from the continuum constitutive model (1) through the imposition of the strong discontinuity kinematics (10), can be summarized as follows:

Free energy $\underbrace{\varphi(\Delta \llbracket \mathbf{u} \rrbracket, \Delta \bar{\alpha})}_{\lim _{h \rightarrow 0}\left(h \psi\left(\varepsilon_{s}, r_{S}\right)\right)}=(1-\omega) \varphi_{0}(\Delta \llbracket \mathbf{u} \rrbracket), \quad\left\{\begin{array}{l}\varphi_{0}(\Delta \llbracket \mathbf{u} \rrbracket)=\frac{1}{2} \Delta \llbracket \mathbf{u} \rrbracket \cdot Q^{\mathrm{e}} \cdot \Delta \llbracket \mathbf{u} \rrbracket, \\ \mathbf{Q}^{\mathrm{e}}=\mathbf{n} \cdot \mathbf{C} \cdot \mathbf{n},\end{array}\right.$

Constitutive equation $\quad \mathscr{T}=\frac{\partial \varphi(\Delta \llbracket \mathbf{u} \rrbracket, \Delta \bar{\alpha})}{\partial \Delta \llbracket \mathbf{u} \rrbracket}, \quad \mathscr{T}=(1-\omega) \mathbf{Q}^{\mathrm{e}} \cdot \Delta \llbracket \mathbf{u} \rrbracket$,

Damage variable $\quad \omega=1-\frac{\bar{q}(\Delta \bar{\alpha})}{\Delta \bar{\alpha}}, \quad \omega \in(-\infty, 1]$,

Evolution law $\frac{\partial}{\partial t}(\Delta \bar{\alpha})=\dot{\bar{\alpha}}=\bar{\lambda}, \quad \Delta \bar{\alpha} \in[0, \infty)$,

Damage criterion $\quad \mathscr{F}(\mathscr{T}, \bar{q}) \equiv \tau_{\mathscr{T}}-\bar{q}, \quad \tau_{\mathscr{T}}=\|\mathscr{T}\|_{\left[\mathbf{Q}^{\mathrm{e}}\right]^{-1}}=\sqrt{\mathscr{T} \cdot\left[\mathbf{Q}^{\mathrm{e}}\right]^{-1} \cdot \mathscr{T}}$,

Loading-unloading conditions $\quad \mathscr{F} \leqslant 0 \quad \bar{\lambda} \geqslant 0 \quad \bar{\lambda} \mathscr{F}=0, \quad \bar{\lambda} \dot{\mathscr{F}}=0$,

Hardening rule $\quad \dot{\bar{q}}=\overline{\mathscr{H}}(\Delta \bar{\alpha}) \dot{\bar{\alpha}} \quad\left(\overline{\mathscr{H}}=\frac{1}{h} \mathscr{H}\right) \quad\left\{\begin{array}{l}\bar{q} \in\left[0, q_{\mathrm{SD}}\right] \\ \left.\bar{q}\right|_{t=t_{\mathrm{SD}}}=q_{\mathrm{SD}}\end{array}\right.$

Remark 8. The model in Eqs. (51a)-(51g) is a discrete damage model characterized by the discrete damage variable $\omega$ in Eq. (51c) (which evolves in terms of the discrete internal variable $\Delta \bar{\alpha}$ ) and the secant (degraded) discrete constitutive modulus $\mathbf{Q}^{\mathrm{s}}=(1-\omega) \mathbf{Q}^{\mathrm{e}}\left(E q\right.$. (51b). Since the initial value of $\omega$ (for $t=t_{\mathrm{SD}}$ and thus $\Delta \bar{\alpha}=0)$ is $\omega=-\infty(\Rightarrow 1-\omega=+\infty)$, the initial secant constitutive modulus is $\mathbf{Q}^{\mathrm{s}}=+\infty \mathbf{Q}^{\mathrm{e}}$ (Fig. 5) and the model can be properly termed as discrete rigid-damage model. 

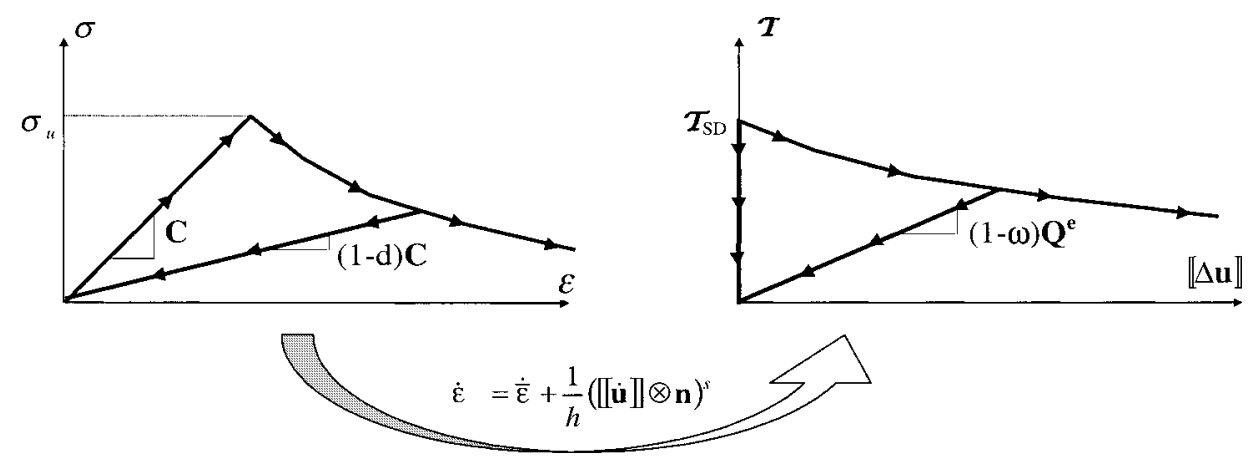

Fig. 5. Damage models: continuum vs. discrete.

\section{Plasticity models}

As a new target, we shall consider now the family of (infinitesimal strain based) plasticity models (Simo and Hughes, 1998) given by

Free energy $\psi\left(\boldsymbol{\varepsilon}, \boldsymbol{\varepsilon}^{\mathrm{p}}, q\right)=\psi^{\mathrm{e}}(\underbrace{\boldsymbol{\varepsilon}-\boldsymbol{\varepsilon}^{\mathrm{p}}}_{\varepsilon^{\mathrm{e}}})+\mathbb{H}(q), \quad \psi^{\mathrm{e}}\left(\boldsymbol{\varepsilon}^{\mathrm{e}}\right)=\frac{1}{2} \varepsilon^{\mathrm{e}}: \mathbf{C}: \boldsymbol{\varepsilon}^{\mathrm{e}}$,

Constitutive equation $\quad \boldsymbol{\sigma}=\partial_{\varepsilon} \psi\left(\boldsymbol{\varepsilon}, \boldsymbol{\varepsilon}^{\mathrm{p}}, q\right), \quad \boldsymbol{\sigma}=\mathbf{C}:\left(\boldsymbol{\varepsilon}-\boldsymbol{\varepsilon}^{\mathrm{p}}\right)$,

Internal variable $\quad \alpha=-\partial_{q} \mathbb{M}(q), \quad \alpha \in[0, \infty)$,

Plastic yield criterion $\quad f(\boldsymbol{\sigma}, q) \equiv \hat{\phi}(\boldsymbol{\sigma})-\left(\sigma_{\mathrm{y}}-q\right)$,

Flow rule $\dot{\boldsymbol{\varepsilon}}^{\mathrm{p}}=\lambda \mathbf{m}, \quad \mathbf{m}(\boldsymbol{\sigma})=\partial_{\boldsymbol{\sigma}} \hat{\phi}(\boldsymbol{\sigma})$,

Evolution law $\dot{\alpha}=\lambda$,

Kuhn-Tucker conditions $\quad f \leqslant 0, \quad \lambda \geqslant 0, \quad \lambda f=0, \quad \lambda \dot{f}=0$,

Hardening rule $\dot{q}=-\mathscr{H} \dot{\alpha}, \quad\left\{\begin{array}{l}q \in\left[0, \sigma_{\mathrm{y}}\right], \\ \mathscr{H}=\left[\partial_{q q}^{2} \mathbb{M}(q)\right]^{-1} \leqslant 0,\end{array}\right.$

where $\psi$ is the free energy and $\psi^{\mathrm{e}}\left(\boldsymbol{\varepsilon}^{\mathrm{e}}\right)$ and, $\mathbb{H}(q)$, its elastic and plastic counterparts, respectively. The total, elastic and plastic strains are denoted, respectively, by $\boldsymbol{\varepsilon}, \boldsymbol{\varepsilon}^{\mathrm{e}}$ and $\boldsymbol{\varepsilon}^{\mathrm{p}}, \mathbf{C}$ is the isotropic elastic constitutive tensor and $q$ is the stress-like (hardening/softening) variable whose thermodynamically conjugated (internal) variable is $\alpha=-\partial_{q} \psi=-\partial_{q} \mathbb{H}(q)$. The yield surface is given in terms of the yield function $f(\boldsymbol{\sigma}, q)$ which defines the elastic domain $\left(\mathrm{E}_{\boldsymbol{\sigma}}:=\left\{(\boldsymbol{\sigma}, q) ; f(\boldsymbol{\sigma}, q) \equiv \hat{\phi}(\boldsymbol{\sigma})-\left(\sigma_{\mathrm{y}}-q\right)<0\right\}\right)$, where $\hat{\phi}(\boldsymbol{\sigma})$ is the equivalent uniaxial stress. The size of the initial elastic domain is given by the initial value of $\left.\left(\sigma_{\mathrm{y}}-q\right)\right|_{t=0}=\sigma_{\mathrm{y}}$ (since $\left.q\right|_{t=0}=0$ ) where $\sigma_{\mathrm{y}}$ is the uniaxial yield stress. The plastic strain evolution (associative) is determined in Eq. (52e) in terms of the plastic multiplier $\lambda$, and the plastic flow tensor $\mathbf{m}\left(\mathbf{m}=\partial_{\sigma} \phi(\boldsymbol{\sigma}) \Rightarrow \mathbf{m}\right.$ is normal to the yield surface $\left.\partial \mathrm{E}_{\sigma}\right)$. Finally, the hardening/softening ${ }^{10}$ parameter, denoted by $\mathscr{H}$ is defined in Eq. (52h).

\footnotetext{
${ }^{10}$ As in the damage model case, it shall be assumed that $\mathscr{H} \leqslant 0$ and, thus, that we are dealing with strain softening.
} 
Remark 9. As for the bounded character of the stress field provided by the constitutive model (52), the same arguments as in Remarks 1 and 3 can be applied here so that both the traction $\mathscr{T}$ and the rate of the traction $\dot{\mathcal{T}}$, as well as the stress field $\boldsymbol{\sigma}_{S}$ and the stress rate $\dot{\boldsymbol{\sigma}}_{S}$ are bounded even for $t \geqslant t_{S D}$. As for the rate $\dot{q}_{S}$ of the hardening/softening variable $q_{S}$, in Eq. (52h), by resorting to the same reasoning than in Remark 3 it can be shown that $\dot{q}_{S} \in\left[0, \dot{\hat{\phi}}\left(\boldsymbol{\sigma}_{S}\right)\right]\left(\dot{q}_{S}=0\right.$ for unloading-neutral loading and $\dot{q}_{S}=\dot{\hat{\phi}}\left(\boldsymbol{\sigma}_{S}\right)$ for loading). On the other hand, $\dot{\hat{\phi}}\left(\boldsymbol{\sigma}_{S}\right)=\partial_{\boldsymbol{\sigma}} \hat{\phi}\left(\boldsymbol{\sigma}_{S}\right): \dot{\boldsymbol{\sigma}}_{S}$ is bounded since both the flow tensor $\mathbf{m}_{S}=\partial_{\boldsymbol{\sigma}} \hat{\phi}\left(\boldsymbol{\sigma}_{S}\right)$ and $\dot{\sigma}_{S}$ are bounded. Therefore, $\dot{q}_{S}$ is bounded even at the strong discontinuity regime when the strains are unbounded.

\section{Strong discontinuity analysis}

\subsection{Discrete hardening law}

For a given point of the discontinuous interface $S$, and from Eq. (52b), we can rewrite the traction continuity condition (12) in the total and rate forms as

$$
\begin{aligned}
& \mathscr{T}=\boldsymbol{\sigma}_{S} \cdot \mathbf{n}=\mathbf{n} \cdot \mathbf{C}:\left(\boldsymbol{\varepsilon}_{S}-\boldsymbol{\varepsilon}_{S}^{\mathrm{p}}\right), \\
& \dot{\mathscr{T}}=\dot{\boldsymbol{\sigma}}_{S} \cdot \mathbf{n}=\mathbf{n} \cdot \mathbf{C}:\left(\dot{\boldsymbol{\varepsilon}}_{S}-\dot{\boldsymbol{\varepsilon}}_{S}^{\mathrm{p}}\right) .
\end{aligned}
$$

Now, for the strong discontinuity regime $\left(t \geqslant t_{\mathrm{SD}}\right.$ and, thus, $\left.h \equiv k \rightarrow 0\right)$ and the strong discontinuity kinematics in Eq. (10)

$$
\begin{aligned}
\dot{\mathscr{T}} & =\lim _{h \rightarrow 0} \mathbf{n} \cdot \mathbf{C}:\left(\dot{\boldsymbol{\varepsilon}}_{S}-\dot{\boldsymbol{\varepsilon}}_{S}^{\mathrm{p}}\right)=\lim _{h \rightarrow 0} \mathbf{n} \cdot \mathbf{C}:\left(\dot{\overline{\boldsymbol{\varepsilon}}}+\frac{1}{h}(\llbracket \dot{\mathbf{u}} \rrbracket \otimes \mathbf{n})^{\mathrm{s}}-\dot{\boldsymbol{\varepsilon}}_{S}^{\mathrm{p}}\right) \\
& =\lim _{h \rightarrow 0}\left(\mathbf{n} \cdot \mathbf{C}: \dot{\overline{\boldsymbol{\varepsilon}}}+\frac{1}{h} \mathbf{n} \cdot \mathbf{C}:(\llbracket \dot{\mathbf{u}} \rrbracket \otimes \mathbf{n})^{\mathrm{s}}-\mathbf{n} \cdot \mathbf{C}: \dot{\boldsymbol{\varepsilon}}_{S}^{\mathrm{p}}\right) \\
& =\lim _{h \rightarrow 0}(\mathbf{n} \cdot \mathbf{C}: \dot{\overline{\boldsymbol{\varepsilon}}}+\frac{1}{h} \underbrace{(\mathbf{n} \cdot \mathbf{C} \cdot \mathbf{n})}_{\mathbf{Q}^{\mathrm{e}}} \cdot \llbracket \dot{\mathbf{u}} \rrbracket-\mathbf{n} \cdot \mathbf{C}: \dot{\boldsymbol{\varepsilon}}_{S}^{\mathrm{p}})
\end{aligned}
$$

where, again, $\mathbf{Q}^{\mathrm{e}}=\mathbf{n} \cdot \mathbf{C} \cdot \mathbf{n}$ is the standard elastic acoustic tensor $\left(\mathbf{Q}^{\mathrm{e}}=(\hat{\lambda}+\mu) \mathbf{n} \otimes \mathbf{n}+\mu \mathbf{1}\right)$ which is nonsingular $\left(\operatorname{det}\left(\mathbf{Q}^{\mathrm{e}}\right)>0\right)$. Some algebraic operations on Eq. (54) lead to

$$
\mathbf{Q}^{\mathrm{e}} \cdot \llbracket \dot{\mathbf{u}} \rrbracket=\lim _{h \rightarrow 0}\left[h(\underbrace{\dot{\mathscr{T}}-\mathbf{n} \cdot \mathbf{C}: \dot{\overline{\boldsymbol{\varepsilon}}}}_{\text {bounded }})+h \mathbf{n} \cdot \mathbf{C}: \dot{\boldsymbol{\varepsilon}}_{S}^{\mathrm{p}}\right]=\mathbf{n} \cdot \mathbf{C}: \lim _{h \rightarrow 0} h \dot{\boldsymbol{\varepsilon}}_{S}^{\mathrm{p}} .
$$

We observe in Eq. (55) that the term over the bracket is bounded (since $\dot{\mathscr{T}}$ is bounded (see Remark 9) and $\dot{\overline{\boldsymbol{\varepsilon}}}$ is bounded by definition) and the corresponding term drops out when $h \rightarrow 0$. Now, we consider two possible situations:

- The plastic flow $\dot{\boldsymbol{\varepsilon}}_{S}^{\mathrm{p}}$ is bounded $\left(\Rightarrow \lim _{h \rightarrow 0} h \dot{\boldsymbol{\varepsilon}}_{S}^{\mathrm{p}}=\mathbf{0}\right)$. Therefore Eq. (55) $\operatorname{reads} \mathbf{Q}^{\mathrm{e}} \cdot \llbracket \dot{\mathbf{u}} \rrbracket=\mathbf{0} \Rightarrow \llbracket \dot{\mathbf{u}} \rrbracket=0$ (since $\mathbf{Q}^{\mathrm{e}}$ is non-singular) and there is no evolution of the discontinuity for $t \geqslant t_{\mathrm{SD}}$ which is in contradiction with the aim of the modeling. Consequently, this situation should be discarded.

- The plastic flow $\dot{\varepsilon}_{S}^{\mathrm{p}}$ is unbounded for $h \rightarrow 0$ ( $\Rightarrow \lim _{h \rightarrow 0} h \dot{\varepsilon}_{S}^{\mathrm{p}}=$ bounded). Since the plastic flow tensor $\mathbf{m}$ is bounded (see Remark 9) from Eq. (52e), we can write

$$
\begin{aligned}
\lim _{h \rightarrow 0} h \dot{\boldsymbol{\varepsilon}}_{S}^{\mathrm{p}} & =\lim _{h \rightarrow 0}\left(h \lambda_{S}\right) \mathbf{m}_{S}=\text { bounded }, \\
& \Rightarrow \lim _{h \rightarrow 0}\left(h \lambda_{S}\right)=\text { bounded. }
\end{aligned}
$$


Now, from Eq. (52f) we define the discrete plastic multiplier $\bar{\lambda} \geqslant 0$ and the discrete internal variable $\bar{\alpha}$ through

$$
\begin{aligned}
& \left\{\begin{array}{l}
\bar{\lambda}=\dot{\bar{\alpha}} \stackrel{\text { def }}{=} h \lambda_{S}=h \dot{\alpha}_{S}=(\text { bounded }), \quad \bar{\alpha} \in[0, \infty) \quad \forall t \geqslant t_{\mathrm{WD}}, \\
\left.\bar{\alpha}\right|_{t=t_{\mathrm{WD}}}=0,
\end{array}\right. \\
& \lambda_{S}=\dot{\alpha}_{S}=\frac{1}{h} \dot{\bar{\alpha}} \quad \forall t \geqslant t_{\mathrm{WD}}
\end{aligned}
$$

that defines the evolution of $\bar{\alpha}$, which will be imposed to be bounded as well as the rate $\dot{\bar{\alpha}}$. The explicit expression of $\alpha_{S}$ in terms of $\bar{\alpha}$ can be obtained for $t \geqslant t_{\mathrm{SD}}$ by integration of Eq. (59) as

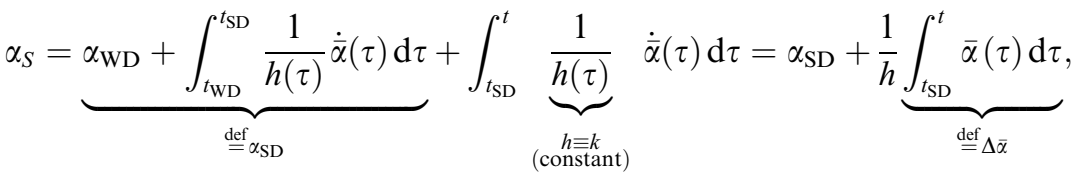

$$
\begin{aligned}
& \Rightarrow\left\{\begin{array}{l}
\alpha_{S}=\alpha_{\mathrm{SD}}+\frac{1}{h} \Delta \bar{\alpha}, \\
\alpha_{\mathrm{SD}}=\left.\alpha_{S}\right|_{t=t_{\mathrm{SD}}}=\alpha_{\mathrm{WD}}+\int_{t_{\mathrm{WD}}}^{t_{\mathrm{SD}}} \frac{1}{h(\tau)} \dot{\bar{\alpha}}(\tau)\left(\Rightarrow \lim _{h \rightarrow 0} h \alpha_{\mathrm{SD}}=0\right), \\
\alpha_{\mathrm{WD}}=\left.\alpha_{S}\right|_{t=t_{\mathrm{WD}}}, \\
\Delta \bar{\alpha}_{t}=\bar{\alpha}_{t}-\bar{\alpha}_{t_{\mathrm{SD}}} \in[0, \infty) .
\end{array}\right.
\end{aligned}
$$

Now, by substituting Eq. (59) into Eq. (52h):

$$
\underbrace{\dot{q}_{S}}_{\text {(bounded) }}=-\mathscr{H} \dot{\alpha}_{S}=-\mathscr{H} \frac{1}{h} \underbrace{\dot{\bar{\alpha}}}_{\text {(bounded) }}
$$

and, thus, consistency of Eq. (62) implies that

$$
\lim _{h \rightarrow 0} \mathscr{H} \frac{1}{h}=\text { bounded }=\overline{\mathscr{H}}
$$

and, as in Eqs. (24)-(27), we recover the concepts of the discrete softening parameter $\overline{\mathscr{H}}$ through the softening parameter regularization condition: ${ }^{11}$

$$
\mathscr{H}(t)=h(t) \overline{\mathscr{H}}, \quad t \geqslant t_{\mathrm{WD}}
$$

and

Discrete softening law $\rightarrow \dot{q}_{S}=-\overline{\mathscr{H}} \dot{\bar{\alpha}} \rightarrow \bar{q}(\Delta \bar{\alpha}) \equiv q_{S}=$

$$
=q_{\mathrm{SD}}+\int_{\bar{\alpha}_{\mathrm{SD}}}^{\bar{\alpha}}-\overline{\mathscr{H}}(\hat{\alpha}) \mathrm{d} \hat{\alpha}, \quad\left\{\begin{array}{l}
\overline{\mathscr{H}}<0, \\
\bar{q} \in\left[0, q_{\mathrm{SD}}\right], \\
\Delta \bar{\alpha} \in[0, \infty) .
\end{array}\right.
$$

Finally, substitution of the flow rule (52e) $\left(\dot{\varepsilon}_{S}^{\mathrm{p}}=\lambda_{S} \mathbf{m}\right)$ and Eq. (59) into Eq. (55) yields

$$
\begin{aligned}
& \mathbf{Q}^{\mathrm{e}} \cdot \llbracket \dot{\mathbf{u}} \rrbracket=\mathbf{n} \cdot \mathbf{C}: \lim _{h \rightarrow 0} h \lambda_{S} \mathbf{m}_{S}=\mathbf{n} \cdot \mathbf{C}: \dot{\bar{\alpha}} \mathbf{m}_{S}=\dot{\bar{\alpha}} \mathbf{n} \cdot \mathbf{C}: \mathbf{m}_{S} \\
& \Rightarrow \llbracket \dot{\mathbf{u}} \rrbracket=\dot{\bar{\alpha}}\left[\mathbf{Q}^{\mathrm{e}}\right]^{-1} \cdot\left[\mathbf{n} \cdot \mathbf{C}: \mathbf{m}\left(\sigma_{S}\right)\right]
\end{aligned}
$$

which provides the evolution of the jump $\llbracket \dot{\mathbf{u}} \rrbracket$ in terms of the stress field $\boldsymbol{\sigma}_{S}$ and $\dot{\bar{\alpha}}$.

\footnotetext{
${ }^{11}$ Again, the discrete softening parameter $\overline{\mathscr{H}}$ is not necessarily constant and a variable value $\mathscr{H}(\bar{\alpha})$ can be considered.
} 


\subsection{Discrete constitutive equation - strong discontinuity conditions}

The results in Section 7.1 are obtained on the basis that the (rate of) traction $\dot{\mathscr{T}}$ is bounded (Eq. (55)). However, these results can be now extended by resorting to the fact that not only the traction rate $\left(\dot{\mathscr{T}}=\dot{\boldsymbol{\sigma}}_{S} \cdot \mathbf{n}\right)$, but also the complete rate of stress $\dot{\boldsymbol{\sigma}}_{S}$ has to remain bounded at the strong discontinuity regime.

Let us then substitute, for a given point of $S$, the kinematics (9) and the flow rule (52e) into the rate form of the constitutive equation (52b):

$$
\dot{\boldsymbol{\sigma}}_{S}=\mathbf{C}:\left(\dot{\boldsymbol{\varepsilon}}_{S}-\dot{\boldsymbol{\varepsilon}}_{S}^{\mathrm{p}}\right)=\mathbf{C}:\left(\dot{\overline{\boldsymbol{\varepsilon}}}+\frac{1}{h}(\llbracket \dot{\mathbf{u}} \rrbracket \otimes \mathbf{n})^{\mathrm{s}}-\lambda_{S} \mathbf{m}_{S}\right)=\mathbf{C}:\left[\overline{\boldsymbol{\varepsilon}}+\frac{1}{h}(\llbracket \dot{\mathbf{u}} \rrbracket \otimes \mathbf{n})^{\mathrm{s}}-\frac{1}{h} \dot{\bar{\alpha}} \mathbf{m}_{S}\right]
$$

where the regularization of the plastic multiplier $\left(\lambda_{S}=\dot{\bar{\alpha}} / h\right)$ in Eq. (59) has been considered. Multiplying both sides of Eq. (68) by $h$ and considering the strong discontinuity regime $\left(t \geqslant t_{\mathrm{SD}} \Rightarrow h \rightarrow 0\right.$ )

$$
\begin{aligned}
& \lim _{h \rightarrow 0} h \dot{\boldsymbol{\sigma}}_{S}=\lim _{h \rightarrow 0} \mathbf{C}:[\underbrace{h \dot{\overline{\boldsymbol{\varepsilon}}}}_{=0}+(\llbracket \dot{\mathbf{u}} \rrbracket \otimes \mathbf{n})^{\mathrm{s}}-\dot{\bar{\alpha}} \mathbf{m}_{S}]=\mathbf{C}:\left[(\llbracket \dot{\mathbf{u}} \rrbracket \otimes \mathbf{n})^{\mathrm{s}}-\dot{\bar{\alpha}} \mathbf{m}_{S}\right] \\
& \Rightarrow(\llbracket \dot{\mathbf{u}} \rrbracket \otimes \mathbf{n})^{\mathrm{s}}-\dot{\bar{\alpha}} \mathbf{m}_{S}=\mathbf{C}^{-1}: \lim _{h \rightarrow 0} h \dot{\boldsymbol{\sigma}}_{S} .
\end{aligned}
$$

Since $\dot{\boldsymbol{\sigma}}_{S}$ is bounded then $\lim _{h \rightarrow 0} h \dot{\boldsymbol{\sigma}}_{S}=0$ and Eq. (69) reads

$$
(\llbracket \dot{\mathbf{u}} \rrbracket \otimes \mathbf{n})^{\mathrm{s}}=\dot{\bar{\alpha}} \mathbf{m}_{S}
$$

which constitutes the strong discontinuity equation. ${ }^{12}$ Like in the damage model case, this is a set of six algebraic equations, relating $\mathbf{m}\left(\boldsymbol{\sigma}_{S}\right)$ and $\llbracket \dot{\mathbf{u}} \rrbracket$, from which we can extract three discrete constitutive equations (relating $\llbracket \dot{\mathbf{u}} \rrbracket$ and $\boldsymbol{\sigma}_{S}$ ) and three additional constraints on the stress field $\boldsymbol{\sigma}_{S}$. By premultiplying Eq. (70) by the symmetric fourth-order unit tensor $\mathbf{I}\left(\mathrm{I}_{i j k l}=1 / 2\left(\delta_{i k} \delta_{j l}+\delta_{i l} \delta_{j k}\right)\right)$ and then by $\mathbf{n}$ we obtain

$$
\left.\begin{array}{c}
\mathbf{I}:(\llbracket \dot{\mathbf{u}} \rrbracket \otimes \mathbf{n})^{\mathrm{s}}=\dot{\bar{\alpha}} \mathbf{I}: \mathbf{m}_{S}=\dot{\bar{\alpha}} \mathbf{m}_{S} \\
\mathbf{n} \cdot \mathbf{I}:(\llbracket \dot{u} \rrbracket \otimes n)^{\mathrm{s}}=\underbrace{(\mathbf{n} \cdot \mathbf{I} \cdot \mathbf{n})}_{\mathbf{Q}^{*}} \cdot \mathbf{Q}^{*} \llbracket \dot{\mathbf{u}} \rrbracket=\mathbf{Q}^{*} \cdot \llbracket \dot{\mathbf{u}} \rrbracket=\dot{\bar{\alpha}} \mathbf{m}_{S} \cdot \mathbf{n} \\
\left(\mathbf{Q}^{*} \stackrel{\operatorname{def}}{=} \mathbf{n} \cdot \mathbf{I} \cdot \mathbf{n}=\frac{1}{2}(\mathbf{n} \otimes \mathbf{n}+\mathbf{1}), \quad\left[\mathbf{Q}^{*}\right]^{-1}=-\mathbf{n} \otimes \mathbf{n}+2 \mathbf{1}\right)
\end{array}\right\} \Rightarrow
$$

which is the discrete constitutive equation. In Eq. (72), $\mathrm{m}^{*}$ is the discrete flow vector whose components, again in the local base $\left(\hat{\mathbf{e}}_{1} \equiv \mathbf{n}, \hat{\mathbf{e}}_{2}, \hat{\mathbf{e}}_{3}\right)$ and after some algebraic manipulation, read

$$
\left[\begin{array}{c}
\mathfrak{m}_{1}^{*} \\
\mathfrak{m}_{2}^{*} \\
\mathfrak{m}_{3}^{*}
\end{array}\right]=\left[\begin{array}{c}
m_{11_{s}} \\
2 m_{12_{S}} \\
2 m_{13_{s}}
\end{array}\right]
$$

and, substitution of Eq. (73) into Eq. (72) yields

\footnotetext{
${ }^{12}$ Notice the formal similarity of this strong discontinuity equation, for plastic models, with the one obtained for damage models (Eq. (40)), the main difference here being that it is given in rate form.
} 


$$
\text { Discrete constitutive equation } \rightarrow\left\{\begin{array}{l}
\llbracket \dot{u} \rrbracket_{1}=\dot{\bar{\alpha}} m_{11_{s}} \\
\llbracket \dot{u} \rrbracket_{2}=\dot{\bar{\alpha}} 2 m_{12_{S}} \\
\llbracket\left[\dot{u} \rrbracket_{3}=\dot{\bar{\alpha}} 2 m_{13_{S}}\right.
\end{array} \quad \forall t \geqslant t_{\mathrm{SD}}\right.
$$

The three additional constraints provided by the strong discontinuity Eq. (70) can be readily obtained from the components of such equation in the aforementioned orthonormal base:

$$
\left[\begin{array}{ccc}
\llbracket \dot{u} \rrbracket_{1} & \frac{1}{2} \llbracket \dot{u} \rrbracket_{2} & \frac{1}{2} \llbracket \dot{u} \rrbracket_{3} \\
\frac{1}{2} \llbracket \dot{u} \rrbracket_{2} & 0 & 0 \\
\frac{1}{2} \llbracket \dot{u} \rrbracket_{3} & 0 & 0
\end{array}\right]=\dot{\bar{\alpha}}\left[\begin{array}{ccc}
m_{11} & m_{12} & m_{13} \\
m_{12} & m_{22} & m_{23} \\
m_{13} & m_{23} & m_{33}
\end{array}\right]_{S}
$$

and noticing that, apart from the discrete constitutive equations (74), Eq. (75) imposes that

Strong discontinuity conditions $\rightarrow m_{22_{s}}=m_{23_{s}}=m_{33_{s}}=0 \quad \forall t \geqslant t_{\mathrm{SD}}$.

\subsection{Discrete yield function - discrete flow rule}

A remarkable consequence of the strong discontinuity conditions (76) is that, in view of the definition of the flow tensor in Eq. (52e), $\left(\mathbf{m}(\sigma)=\partial_{\sigma} \hat{\phi}(\boldsymbol{\sigma})\right)$, one can write

$$
\left.\begin{array}{l}
\partial_{\sigma_{22}} \hat{\phi}\left(\boldsymbol{\sigma}_{s}\right)=m_{22_{s}}=0 \\
\partial_{\sigma_{23}} \hat{\phi}\left(\boldsymbol{\sigma}_{s}\right)=m_{23_{s}}=0 \\
\partial_{\sigma_{23}} \hat{\phi}\left(\boldsymbol{\sigma}_{s}\right)=m_{33_{s}}=0
\end{array}\right\} \quad \forall t \geqslant t_{\mathrm{SD}},
$$

so that, during the strong discontinuity regime, there is no dependence of the equivalent uniaxial stress $\hat{\phi}\left(\boldsymbol{\sigma}_{s}\right)$ on the components $\sigma_{22}, \sigma_{23}$ and $\sigma_{33}$. Therefore, $\hat{\phi}\left(\boldsymbol{\sigma}_{s}\right)$ is a function of the remaining components $\boldsymbol{\sigma}_{s}$ only, i.e., those defining the traction $\mathscr{T}$ :

$$
\hat{\phi}\left(\boldsymbol{\sigma}_{s}\right)=\hat{\phi}(\underbrace{\{\sigma_{11}, \overbrace{\sigma_{12}}^{=\sigma_{21}}, \overbrace{\sigma_{13}}^{=\sigma_{31}}\}}_{\{\mathscr{T}\}^{T}})=F(\mathscr{T})
$$

and, then, the yield function (52d) considering Eqs. (65) and (78), reads

Discrete yield function $\rightarrow f\left(\boldsymbol{\sigma}_{S}, q\right)=\hat{\phi}\left(\boldsymbol{\sigma}_{S}\right)-\left(\sigma_{\mathrm{y}}-q_{S}\right) \equiv \mathscr{F}(\mathscr{T})-\left(\sigma_{\mathrm{y}}-\bar{q}(\Delta \bar{\alpha})\right) \stackrel{\text { def }}{=} F(\mathscr{T}, \bar{q})$

which constitutes the discrete yield function. Besides, the discrete constitutive Eqs. (72)-(74) can be expressed as a discrete flow rule in terms of the discrete plastic multiplier $\bar{\lambda}=\dot{\bar{\alpha}}$ defined in Eq. (58):

Discrete flow rule $\rightarrow\left\{\begin{array}{l}\llbracket \dot{\mathbf{u}} \rrbracket=\dot{\bar{\alpha}} \mathrm{m}^{*}=\bar{\lambda}, \quad \mathrm{m}^{*}(\mathscr{T})=\partial \mathscr{T} F(\mathscr{T}), \\ {\left[\mathrm{m}^{*}\right]=\left[\begin{array}{c}m_{11} \\ 2 m_{12} \\ 2 m_{13}\end{array}\right]_{\mathscr{S}}=\left[\begin{array}{c}\frac{\partial \hat{\phi}}{\partial \sigma_{11}} \\ \frac{\partial \hat{\phi}}{\partial \sigma_{12}}+\frac{\partial \hat{\phi}}{\partial \sigma_{11}} \\ \frac{\partial \hat{\phi}}{\partial \sigma_{13}}+\frac{\partial \hat{\phi}}{\partial \sigma_{31}}\end{array}\right]_{\mathscr{S}}=\left[\begin{array}{l}\frac{\partial F}{\partial \mathscr{T}} \\ \frac{\partial F}{\partial \mathscr{T}_{2}} \\ \frac{\partial F}{\partial \mathscr{T}_{3}}\end{array}\right] .}\end{array}\right.$

\subsection{Discrete elasto-plastic free energy}

Now, we notice that the strong discontinuity equation (70) yields

$$
(\llbracket \dot{\mathbf{u}} \rrbracket \otimes \mathbf{n})^{\mathrm{s}}=\underbrace{\dot{\bar{\alpha}}}_{\lambda_{S} h} \mathbf{m}_{S}=\lambda_{S} h \mathbf{m}_{S} \Rightarrow \frac{1}{h}(\llbracket \dot{\mathbf{u}} \rrbracket \otimes \mathbf{n})^{\mathrm{s}}=\lambda_{S} \mathbf{m}_{S}=\dot{\boldsymbol{\varepsilon}}_{S}^{\mathrm{p}},
$$


where Eq. (58) and the continuum flow rule definition (52e) have been considered. Eq. (81) states that at the strong discontinuity regime $\left(t \geqslant t_{\mathrm{SD}}\right)$ the plastic flow translates entirely into displacement jump. Therefore, from Eq. (81) and the strong discontinuity kinematics (9) the (rate of) the elastic strain $\dot{\boldsymbol{\varepsilon}}_{S}^{\mathrm{e}}$ reads

$$
\dot{\boldsymbol{\varepsilon}}_{S}^{\mathrm{e}}=\dot{\boldsymbol{\varepsilon}}_{S}-\dot{\boldsymbol{\varepsilon}}_{S}^{\mathrm{p}}=\dot{\overline{\boldsymbol{\varepsilon}}}+\underbrace{\frac{1}{h}(\llbracket \dot{\mathbf{u}} \rrbracket \otimes \mathbf{n})^{\mathrm{s}}}_{\dot{\varepsilon}_{S}^{\mathrm{p}}}-\dot{\boldsymbol{\varepsilon}}_{S}^{\mathrm{p}}=\dot{\overline{\boldsymbol{\varepsilon}}} \quad \forall t \geqslant t_{\mathrm{SD}}
$$

Eq. (82) can be integrated along time leading to

$$
\begin{aligned}
& \boldsymbol{\varepsilon}_{S}^{\mathrm{e}}=\int_{0}^{t} \dot{\boldsymbol{\varepsilon}}_{S}^{\mathrm{e}}(\tau) \mathrm{d} \tau=\underbrace{\int_{0}^{t_{\mathrm{SD}}} \dot{\boldsymbol{\varepsilon}}_{S}^{\mathrm{e}}(\tau) \mathrm{d} \tau}_{\varepsilon_{\mathrm{SD}}^{\mathrm{e}}}+\int_{t_{\mathrm{SD}}}^{t} \underbrace{\dot{\boldsymbol{\varepsilon}}_{S}^{\mathrm{e}}(\tau)}_{\overline{\bar{\varepsilon}}} \mathrm{d} \tau=\boldsymbol{\varepsilon}_{\mathrm{SD}}^{\mathrm{e}}+\Delta \overline{\boldsymbol{\varepsilon}} \\
& \left(\begin{array}{l}
\left.\boldsymbol{\varepsilon}_{\mathrm{SD}}^{\mathrm{e}} \stackrel{\text { def }}{=} \int_{0}^{t \mathrm{SD}} \dot{\boldsymbol{\varepsilon}}_{S}^{\mathrm{e}}(\tau) \mathrm{d} \tau \Rightarrow \lim _{h \rightarrow 0} h \boldsymbol{\varepsilon}_{\mathrm{SD}}^{\mathrm{e}}=\mathbf{0}\right) \\
\left(\Delta \overline{\boldsymbol{\varepsilon}}^{\text {def }} \overline{\boldsymbol{\varepsilon}}_{t}-\overline{\boldsymbol{\varepsilon}}_{\mathrm{SD}} \Rightarrow \lim _{h \rightarrow 0} h \Delta \overline{\boldsymbol{\varepsilon}}=\mathbf{0}\right)
\end{array}\right\} \Rightarrow \lim _{h \rightarrow 0} h \boldsymbol{\varepsilon}_{S}^{\mathrm{e}}=\mathbf{0} .
\end{aligned}
$$

Let us now consider, at a given material point of the interface $S$, the continuum free energy (see Eq. $(52 a))$ :

$$
\psi\left(\varepsilon_{S}, \varepsilon_{S}^{\mathrm{p}}, q_{S}\right)=\psi^{\mathrm{e}}\left(\varepsilon_{S}^{\mathrm{e}}\right)+\mathbb{W}\left(q_{S}\right)
$$

which provides, for the continuum model (52), the stresses $\sigma_{S}$ and the internal variable $\alpha_{S}$ as conjugate variables, respectively, of the strains $\boldsymbol{\varepsilon}_{S}$ and the hardening/softening variable $q_{S}$ (see Eqs. (52b) and (52c)). For the latter, and considering the definition $\Delta \bar{\alpha}=h\left(\alpha_{S}-\alpha_{\mathrm{SD}}\right.$ ) (from Eq. (61))

$$
\alpha_{S}=-\partial_{q_{S}} \psi\left(\varepsilon_{S}, \varepsilon_{S}^{\mathrm{p}}, q_{S}\right) \Rightarrow \Delta \bar{\alpha}=h\left(\alpha_{S}-\alpha_{\mathrm{SD}}\right)=-h \partial_{q_{S}} \psi\left(\varepsilon_{S}, \varepsilon_{S}^{\mathrm{p}}, q_{S}\right)-h \alpha_{\mathrm{SD}}
$$

and for the strong discontinuity regime ( $t \geqslant t_{\mathrm{SD}}$ and, thus, $h=k \rightarrow 0$ )

$$
\Delta \bar{\alpha}=-\lim _{h \rightarrow 0}(h \partial_{q_{S}} \psi\left(\boldsymbol{\varepsilon}_{S}, \boldsymbol{\varepsilon}_{S}^{\mathrm{p}}, \mathbf{q}_{S}\right)-\underbrace{h \alpha_{\mathrm{SD}}}_{=0})=-\partial_{q_{S}} \underbrace{\lim _{h \rightarrow 0} h \psi\left(\boldsymbol{\varepsilon}_{S}, \boldsymbol{\varepsilon}_{S}^{\mathrm{p}}, q_{S}\right)}_{\substack{\text { def } \\ \underbrace{h \rightarrow 0}_{\varphi}}}=-\partial_{\bar{q}} \varphi,
$$

where Eq. (61) $\left(\lim _{h \rightarrow 0} h \alpha_{\mathrm{SD}}=0\right)$ has been considered. Eq. (86) defines, from the continuum free energy $\psi$ in Eq. (84), the discrete elasto-plastic free energy $\varphi$ as

$$
\varphi(\bar{q})=\lim _{h \rightarrow 0} h \psi\left(\boldsymbol{\varepsilon}_{S}, \boldsymbol{\varepsilon}_{S}^{\mathrm{p}}, q_{S}\right)=\lim _{h \rightarrow 0}\left[h \psi^{\mathrm{e}}\left(\boldsymbol{\varepsilon}_{S}^{\mathrm{e}}\right)+h \llbracket(\underbrace{q_{S}}_{=\bar{q}})\right]=\underbrace{\lim _{h \rightarrow 0} \frac{1}{2} h \boldsymbol{\varepsilon}_{S}^{\mathrm{e}}: \mathbf{C}: \boldsymbol{\varepsilon}_{S}^{\mathrm{e}}}_{=0}+\underbrace{\lim _{h \rightarrow 0} h \llbracket\left(q_{S}\right)}_{\stackrel{\text { def }}{=} \mathrm{H}(\overline{\mathbf{q}})}=\mathbf{H}(\bar{q})
$$

Discrete free energy $\rightarrow \varphi(\bar{q})=\lim _{h \rightarrow 0} h \psi\left(\boldsymbol{\varepsilon}_{S}, \boldsymbol{\varepsilon}_{S}^{\mathrm{p}}, q_{S}\right)=\lim _{h \rightarrow 0} h \llbracket\left(q_{S}\right)=\mathrm{H}(\bar{q})$

where the definition (52a) of the elastic free energy, $\psi^{\mathrm{e}}\left(\boldsymbol{\varepsilon}^{\mathrm{e}}\right)=\frac{1}{2} \boldsymbol{\varepsilon}^{\mathrm{e}}: \mathbf{C}: \boldsymbol{\varepsilon}^{\mathrm{e}}$, and Eq. (83) $\left(\lim _{h \rightarrow 0} h \boldsymbol{\varepsilon}_{S}^{\mathrm{e}}=\mathbf{0}\right)$ have been considered. Notice again, from Eq. (88), that $\varphi$ and $\mathrm{H}$ are both energies per unit surface of discontinuity interface $(=h \times$ energy density $)$.

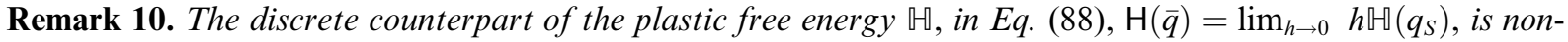
zero and bounded. For instance, for a linear continuum softening law, we have $\mathbb{\square}(q)=\frac{1}{2}\left(q^{2} / \mathscr{H}\right)$. Regularization of the continuum softening parameter $\mathscr{H}$, in Eq. (64) $(\overline{\mathscr{H}}=h \mathscr{H})$, leads to $\mathbb{U}\left(q_{S}\right)=$ $\frac{1}{2}\left(\bar{q}^{2} / h \overline{\mathscr{H}}\right) \Rightarrow \mathrm{H}(\bar{q})=h \llbracket\left(q_{S}\right)=\frac{1}{2}\left(\bar{q}^{2} / \overline{\mathscr{H}}\right) \rightarrow$ non-zero and bounded. 


\section{Discrete elasto-plastic model}

The discrete model obtained in the preceding section can be summarized as follows:

Free energy $\underbrace{\varphi\left(\Delta \llbracket \mathbf{u} \rrbracket^{\mathrm{e}}, \bar{q}\right)}_{\lim _{h \rightarrow 0} h \psi\left(\varepsilon_{S},,_{S}^{\mathrm{p}}, q_{S}\right)}=\varphi^{\mathrm{e}}\left(\Delta \llbracket \mathbf{u} \rrbracket^{\mathrm{e}}\right)+\mathrm{H}(\bar{q}), \quad\left\{\begin{array}{l}\varphi^{\mathrm{e}}\left(\Delta \llbracket \mathbf{u} \rrbracket^{\mathrm{e}}\right)=0, \\ \mathrm{H}(\bar{q})=\lim _{h \rightarrow 0} h \llbracket\left(q_{S}\right),\end{array}\right.$

Internal variable $\quad \Delta \bar{\alpha}=-\partial_{\bar{q}} \varphi(\Delta \llbracket \mathbf{u} \rrbracket, \bar{q})=-\partial_{\bar{q}} \mathrm{H}(\bar{q}), \quad \Delta \bar{\alpha} \in[0, \infty)$,

Plastic yield criterion $\quad \mathscr{F}(\mathscr{T}, q) \equiv F(\mathscr{T})-\left(\sigma_{\mathrm{y}}-\bar{q}(\Delta \bar{\alpha})\right), \quad F(\mathscr{T})=\lim _{h \rightarrow 0} \hat{\phi}\left(\boldsymbol{\sigma}_{s}\right)$,

Plastic flow $\quad \Delta \llbracket \dot{\mathbf{u}} \rrbracket=\llbracket \dot{\mathbf{u}} \rrbracket=\left(\Delta \llbracket \dot{\mathbf{u}} \rrbracket^{\mathrm{p}}\right)=\bar{\lambda} \mathrm{m}^{*}, \quad \mathrm{~m}^{*}(\mathscr{T})=\partial_{\mathscr{T}} F(\mathscr{T})$,

Evolution law $\quad \frac{\partial}{\partial t}(\Delta \bar{\alpha})=\dot{\bar{\alpha}}=\bar{\lambda}, \quad \Delta \bar{\alpha} \in[0, \infty)$,

Kuhn-Tucker conditions $\quad \mathscr{F} \leqslant 0, \bar{\lambda} \geqslant 0, \bar{\lambda} \mathscr{F}=0, \quad \bar{\lambda} \dot{\mathscr{F}}=0$,

Hardening rule $\quad \dot{\bar{q}}=-\overline{\mathscr{H}}(\Delta \bar{\alpha}) \dot{\bar{\alpha}}, \quad \overline{\mathscr{H}}=\frac{1}{h} \mathscr{H}=\left[\partial_{\bar{q}}^{2} \mathrm{H}(\bar{q})\right]^{-1}, \quad\left\{\begin{array}{l}\bar{q} \in\left[q_{\mathrm{SD}}, \sigma_{\mathrm{y}}\right], \\ \left.\bar{q}\right|_{t=t_{\mathrm{SD}}}=q_{\mathrm{SD}} .\end{array}\right.$

Remark 11. The discrete elasto-plastic model (89a)-(89g) has null elastic free energy $\left(\varphi^{\mathrm{e}}\left(\Delta \llbracket \mathbf{u} \rrbracket^{\mathrm{e}}\right)\right)$ (see Eq. (89a)). Owing to this, in the context of an additive decomposition of the displacement jump $\Delta \llbracket \mathbf{u} \rrbracket$ into its elastic and plastic counterparts $\left(\Delta \llbracket \mathbf{u} \rrbracket=\Delta \llbracket \mathbf{u} \rrbracket^{\mathrm{e}}+\Delta \llbracket \mathbf{u} \rrbracket^{\mathrm{p}}\right)$, it can be considered that $\Delta \llbracket \mathbf{u} \rrbracket^{\mathrm{e}}=\mathbf{0}$ and, thus, $\Delta \llbracket \mathbf{u} \rrbracket=\Delta \llbracket \mathbf{u} \rrbracket^{\mathrm{p}}$. This implies that the jump developed at the strong discontinuity regime, $\Delta \llbracket \mathbf{u} \rrbracket$, is totally irreversible upon unloading. Consequently, we are dealing with a rigid-plastic discrete model (see Fig. 6).

It is worth noting the following one to one correspondence between the continuum and the discrete variables that can be extracted from the comparison of the original continuum (Eq. (52)) and the induced discrete (Eqs. (89a)-(89g)) models
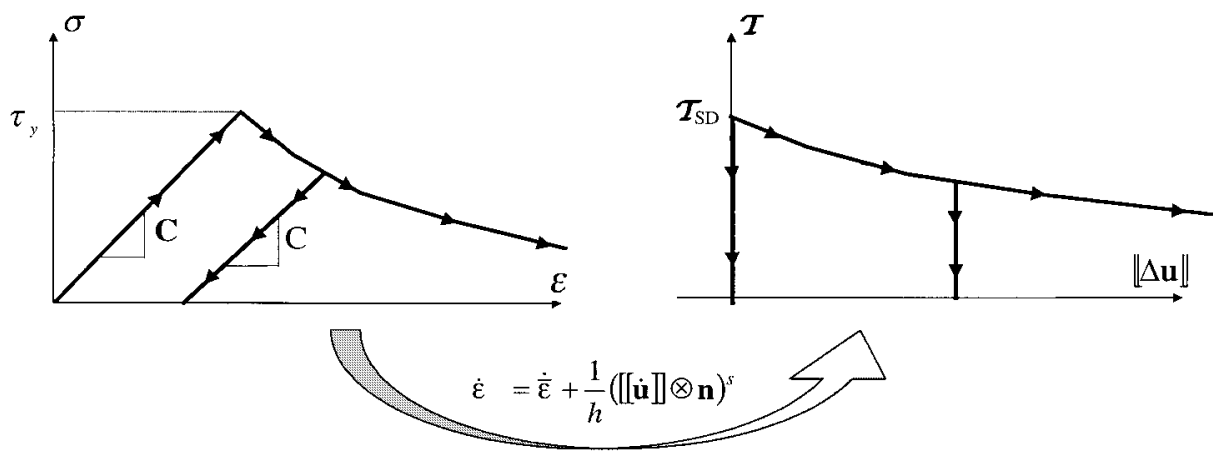

Fig. 6. Elasto-plastic models: continuum vs. discrete. 


\begin{tabular}{||l||c|c|c|c|c||}
\hline \hline Continuum: & $\psi=\psi^{e}\left(\varepsilon^{e}\right)+\mathbb{H}(q)$ & $\boldsymbol{\sigma}$ & $\boldsymbol{\varepsilon}$ & $\boldsymbol{\varepsilon}^{e}$ & $\boldsymbol{\varepsilon}^{p}$ \\
\hline Discrete: & $\varphi=0+\mathbb{H}(\bar{q})$ & $\mathcal{T}$ & $\Delta \llbracket \mathbf{u} \rrbracket$ & $\mathbf{0}$ & $\Delta \llbracket \mathbf{u} \rrbracket$ \\
\hline
\end{tabular}

\begin{tabular}{||l||c|c|c|c|c||}
\hline \hline Continuum: & $\alpha$ & $f(\boldsymbol{\sigma}, q$ & $\mathbf{m}(\boldsymbol{\sigma})=\partial_{\sigma} f$ & $q(\alpha)$ & $\mathcal{H}$ \\
\hline Discrete: & $\Delta \bar{\alpha}$ & $\mathcal{F}(\mathcal{T}, \bar{q})$ & $\mathbf{m}^{*}(\mathcal{T})=\partial_{\mathcal{T}} \mathcal{F}$ & $\bar{q}(\Delta \bar{\alpha})$ & $\overline{\mathcal{H}}$ \\
\hline
\end{tabular}

\section{Concluding remarks}

Throughout the previous sections, a methodology to derive discrete constitutive models from the original continuum ones has been explored. The keypoint of the analysis is to realize that, from the traction continuity (Eq. (12)), both the stress and the rate of the stress fields have to remain bounded at the discontinuous interface even at the strong discontinuity regime when the strains (described from Eq. (10)) become unbounded.

Then, the consistency analysis of the mathematical expressions defining the continuum model (strong discontinuity analysis) shows the softening regularization condition (25) as a sufficient requirement to make them compatible with the appearance of strong discontinuities. In fact, for modeling purposes, this regularization of the continuum softening parameter $\mathscr{H}$ in terms of the discrete one $\overline{\mathscr{H}}(\mathscr{H}=h \overline{\mathscr{H}})$ is the only modification to be done in the standard continuum models to make them available for simulation of strong discontinuities. This is a crucial advantage to be exploited in modeling environments.

Under such conditions, it has also been shown that not only a discrete constitutive equation, but also $a$ complete discrete constitutive model is then induced by the continuum one via the introduction of the strong discontinuity kinematics. At this point, three remarks can be made

(1) Unlike what could have been expected, there is no necessity to resort to anisotropic continuum models to induce the anisotropic (directional) behavior associated to the discrete constitutive ones (as an example, both target models considered in this paper are clearly isotropic). In fact, we could consider the induced discrete models (51a)-(51g) and (89a)-(89g) as directional projections of the continuum ones, these projections being given by the strong discontinuity kinematics which have clear directional ingredients (the normal $\mathbf{n}$ in Eqs. (9) and (10)). So, it is the projection and not the original continuum model that is responsible for the resulting directionality of the induced discrete model.

(2) Actually, the resulting discrete models, which have been fully derived here for the sake of clarity, do not need to be derived and implemented in a computational simulation tool. Once the softening parameter is regularized (as in Eq. (25) or Eq. (64)) and the strong discontinuity kinematics (10) is allowed to develop, the whole formulation can be held in the continuum formalism. The parent continuum model behaves as the induced discrete one would do in a discrete formalism. The advantages and possibilities offered, for simulation purposes, by this approach have already been stated elsewhere (Oliver, 1996a,b; Oliver et al., 1999).

(3) A common feature of the induced discrete models is that they inherit the nature of the continuum ones but specializing them to the rigid case. Indeed, for the models considered in this paper, we have seen that from scalar continuum damage and continuum elasto-plastic models, scalar rigid-damage and rigidplastic discrete models are, respectively, induced (see Remarks 8 and 11). This seems to be a property that can be generalized to other families of constitutive models. 
Unlike what has been frequently done in strong discontinuity analyses of continuum constitutive models, here a full exploitation of the bounded character of the stress field $\boldsymbol{\sigma}_{S}$ (and also of the rate of stress field $\dot{\boldsymbol{\sigma}}_{S}$ ) at the discontinuous interface has been performed, since it has not been restricted to the tractions $\mathscr{T}=\sigma_{S} \cdot \mathbf{n}$. In consequence, on top of the discrete constitutive equations, the additional strong discontinuity conditions have been derived for both the considered families. Such conditions, not always identified in this type of analyses, pose strong restrictions on the induction of strong discontinuities by direct bifurcation of the stress-strain field. Therefore, some transition mechanisms have to be specifically devised (Oliver et al., 1999).

In summary, the proposed methodology when combined with appropriated numerical techniques is envisaged as a possible setting to unify the continuum techniques based on the strain localization phenomena and the discrete ones based on the non-linear decohesive fracture mechanics.

\section{References}

Armero, F., 1997. Localized Anisotropic Damage of Brittle Materials. In: Owen, D., Onate, E., Hinton, E. (Eds.), Computational Plasticity: Fundamentals and Applications, pp. 635-640.

Armero, F., Garikipati, K., 1996. An analysis of strong discontinuities in multiplicative finite strain plasticity and their relation with the numerical simulation of strain localization in solids. Int. J. Solids Struct. 33 (20-22), 2863-2885.

Bazant, Z., Planas, J., 1998. Fracture and Size Effect in Concrete and Other Quasibrittle Materials. CRC Press, Boca Raton, FL.

deBorst, R., Sluys, L.J., Muhlhaus, H.B., Pamin, J., 1993. Fundamental issues in finite element analyses of localization of deformation. Engng. Computat. 10, 99-121.

Larsson, R., Runesson, K., Sture, S., 1996. Embedded localization band in undrained soil based on regularized strong discontinuity theory and finite element analysis. Int. J. Solids Struct. 33 (20-22), 3081-3101.

Oliver, J., 1995a. Continuum Modelling of Strong Discontinuities in Solid Mechanics. In: Owen, D.R.J., Onate, E. (Eds.), Computational Plasticity: Fundamentals and Applications, vol. 1. Pineridge Press, Swansea, pp. 455-479.

Oliver, J., 1995b. Continuum modelling of strong discontinuities in solid mechanics using damage models. Computat. Mech. 17 (1-2), 49-61.

Oliver, J., 1996a. Modeling strong discontinuities in solid mechanics via strain softening constitutive equations, Part 1: Fundamentals. Int. J. Num. Meth. Engng. 39 (21), 3575-3600.

Oliver, J., 1996b. Modeling strong discontinuities in solid mechanics via strain softening constitutive equations, Part 2: Numerical simulation. Int. J. Num. Meth. Engng. 39 (21), 3601-3623.

Oliver, J., 1998. The Strong Discontinuity Approach: an overview. In: Idelsohn, S., Oñate, E., Dvorkin E. (Eds.), Computational Mechanics: New Trends and Applications. WCCM98 Proc. (CD-ROM) IV World Cong. on Computat. Mech., CIMNE. Barcelona, Spain.

Oliver, J., Cervera, M., Manzoli, O., 1997. On the use of J2 Plasticity Models for the Simulation of 2D Strong Discontinuities in Solids block. In: Owen, D., Onate, E., Hinton, E. (Eds.), Proc. Int. Conf. on Computat. Plasticity, Barcelona, Spain, CIMNE, pp. $38-55$.

Oliver, J., Cervera, M., Manzoli, O., 1998. On the Use of Strain-softening Models for the Simulation of Strong Discontinuities in Solids. In: de Borst, R., van der Giessen, E. (Eds.), Material Instabilities in Solids. Wiley, New York, pp.107-123 (chapter 8).

Oliver, J., Cervera, M., Manzoli, O., 1999. Strong discontinuities and continuum plasticity models: the strong discontinuity approach. Int. J. Plasticity 15 (3), 319-351.

Oliver, J., Cervera, M., Oller, S., Lubliner, J., 1990. Isotropic damage models and smeared crack analysis of concrete. In: Bicanic, N., et al. (Eds.), Proc. SCI-C Comput. Aided Anal. and Des. of Concrete Struct, pp. 945-957.

Oliver, J., Simo, J., 1994. Modelling Strong Discontinuities by Means of Strain Softening Constitutive Equations. In: Mang, H., et al. (Eds.), Proc. EURO-C 1994 Comput. Modeling of Concrete Struct. Pineridge Press, Swansea, pp. 363-372.

Ottosen, N., Runesson, K., 1991. Properties of discontinuous bifurcation solutions in elasto-plasticity. Int. J. Solids Struct. 27 (4), 401421.

Runesson, K., Ottosen, N.S., Peric, D., 1991. Discontinuous bifurcations of elastic-plastic solutions at plane stress and plane strain. Int. J. Plasticity 7, 99-121.

Runesson, K., Peric, D., Sture, S., 1996. Effect of pore fluid compressibility on localization in elastic-plastic porous solids under undrained conditions. Int. J. Solids Struct. 33 (10), 1501-1518.

Simo, J., Hughes, T., 1998. Computational Inelasticity. Springer, Berlin.

Simó, J., Ju, J., 1987. Strain and stress based continuum damage models. I. Formulation. Int. J. Solids Struct. 23, 821-840. 
Simo, J., Oliver, J., 1994. A new approach to the analysis and simulation of strong discontinuities. In: Z.B., et al. (Eds.), Fracture and Damage in Quasi-brittle Structures. E\&FN Spon, pp. 25-39.

Simo, J., Oliver, J., Armero, F., 1993. An analysis of strong discontinuities induced by strain-softening in rate-independent inelastic solids. Computat. Mech. 12, 277-296.

Willam, K., Sobh, N., 1987. Bifurcation Analysis of Tangential Material Operators. In: Pande, G.N., Middleton, J. (Eds.), Transient/ Dynamic Analysis and Constitutive Laws for Engineering Materials, vol. 2. Martinus-Nijhoff, The Hague, pp. C4/1-13. 